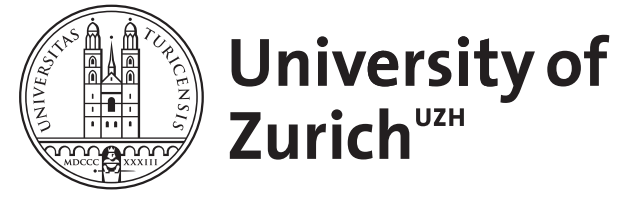

\title{
The cosmic code comparison project
}

Heitmann, K ; Lukić, Z ; Fasel, P ; Habib, S ; Warren, M S ; White, M ; Ahrens, J ; Ankeny, L ; Armstrong, R ; O'Shea, B ; Ricker, P M ; Springel, V ; Stadel, J ; Trac, H

\begin{abstract}
Current and upcoming cosmological observations allow us to probe structures on smaller and smaller scales, entering highly nonlinear regimes. In order to obtain theoretical predictions in these regimes, large cosmological simulations have to be carried out. The promised high accuracy from observations makes the simulation task very demanding: the simulations have to be at least as accurate as the observations. This requirement can only be fulfilled by carrying out an extensive code verification program. The first step of such a program is the comparison of different cosmology codes including gravitational interactions only. In this paper, we extend a recently carried out code comparison project to include five more simulation codes. We restrict our analysis to a small cosmological volume which allows us to investigate properties of halos. For the matter power spectrum and the mass function, the previous results hold, with the codes agreeing at the $10 \%$ level over wide dynamic ranges. We extend our analysis to the comparison of halo profiles and investigate the halo count as a function of local density. We introduce and discuss ParaView as a flexible analysis tool for cosmological simulations, the use of which immensely simplifies the code comparison task.
\end{abstract}

DOI: https://doi.org/10.1088/1749-4699/1/1/015003

Posted at the Zurich Open Repository and Archive, University of Zurich ZORA URL: https://doi.org/10.5167/uzh-16769

Journal Article

Originally published at:

Heitmann, K; Lukić, Z; Fasel, P; Habib, S; Warren, M S; White, M; Ahrens, J; Ankeny, L; Armstrong, R; O'Shea, B; Ricker, P M; Springel, V; Stadel, J; Trac, H (2008). The cosmic code comparison project. Computational Science Discovery, 1(1):015003.

DOI: https://doi.org/10.1088/1749-4699/1/1/015003 
The cosmic code comparison project

This article has been downloaded from IOPscience. Please scroll down to see the full text article.

2008 Comput. Sci. Disc. 1015003

(http://iopscience.iop.org/1749-4699/1/1/015003)

View the table of contents for this issue, or go to the journal homepage for more

Download details:

IP Address: 138.188.100.226

The article was downloaded on 12/03/2011 at 15:38

Please note that terms and conditions apply. 


\title{
com SCIENCE\&DISCOVERY
}

\section{The cosmic code comparison project}

\author{
Katrin Heitmann ${ }^{1}$, Zarija Lukic ${ }^{2}$, Patricia Fasel ${ }^{1}$, Salman Habib ${ }^{1}$, \\ Michael S Warren ${ }^{1}$, Martin White ${ }^{3}$, James Ahrens ${ }^{1}$, Lee Ankeny ${ }^{1}$, \\ Ryan Armstrong ${ }^{4}$, Brian O'Shea ${ }^{1}$, Paul M Ricker ${ }^{2,5}$, Volker Springel ${ }^{6}$, \\ Joachim Stadel ${ }^{7}$ and $\mathrm{Hy} \mathrm{Trac}^{8}$ \\ ${ }^{1}$ Los Alamos National Laboratory, Los Alamos, NM 87545, USA \\ 2 Department of Astronomy, University of Illinois, Urbana, IL 61801, USA \\ ${ }^{3}$ Department of Astronomy, University of California, Berkeley, CA 94720-3411, USA \\ ${ }^{4}$ Department of Computer Science, UC Davis, Davis, CA 95616, USA \\ ${ }^{5}$ National Center for Supercomputing Applications, Urbana, IL 61801, USA \\ ${ }^{6}$ Max-Planck-Institute for Astrophysics, 85741 Garching, Germany \\ ${ }^{7}$ Institute of Theoretical Physics, University of Zurich, 8057 Zurich, Switzerland \\ ${ }^{8}$ Department of Astrophysical Sciences, Princeton University, NJ 08544, USA \\ E-mail: heitmann@lanl.gov
}

Received 23 July 2007, in final form 14 May 2008

Published 20 November 2008

Computational Science \& Discovery 1 (2008) 015003 (24pp)

doi:10.1088/1749-4699/1/1/015003

\begin{abstract}
Current and upcoming cosmological observations allow us to probe structures on smaller and smaller scales, entering highly nonlinear regimes. In order to obtain theoretical predictions in these regimes, large cosmological simulations have to be carried out. The promised high accuracy from observations makes the simulation task very demanding: the simulations have to be at least as accurate as the observations. This requirement can only be fulfilled by carrying out an extensive code verification program. The first step of such a program is the comparison of different cosmology codes including gravitational interactions only. In this paper, we extend a recently carried out code comparison project to include five more simulation codes. We restrict our analysis to a small cosmological volume which allows us to investigate properties of halos. For the matter power spectrum and the mass function, the previous results hold, with the codes agreeing at the $10 \%$ level over wide dynamic ranges. We extend our analysis to the comparison of halo profiles and investigate the halo count as a function of local density. We introduce and discuss ParaView as a flexible analysis tool for cosmological simulations, the use of which immensely simplifies the code comparison task.
\end{abstract}




\section{Contents}

1. Introduction 2

2. The codes $\quad 3$

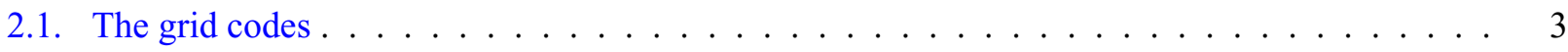

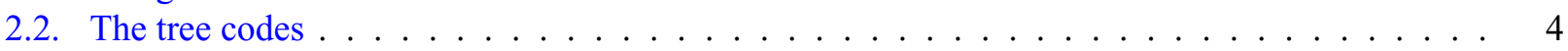

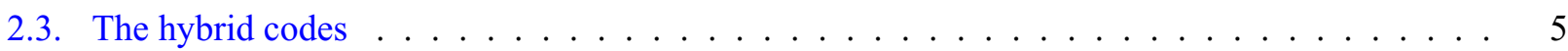

3. The simulations $\quad 6$

$\begin{array}{ll}\text { 4. Analysis framework and tools } & 7\end{array}$

5. Results 9

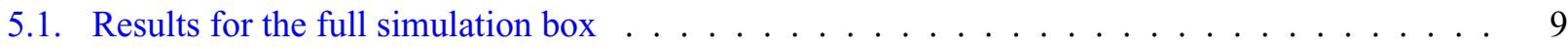

5.2. Dark matter halos . . . . . . . . . . . . . . . . . . . . . . . . 9 9

5.3. The mass function and halo counts as a function of density . . . . . . . . . . . . . . 14

5.4. The power spectrum . . . . . . . . . . . . . . . . . . . . . . 19

6. Discussion and conclusion $\quad 22$

Acknowledgments $\quad 22$

References $\quad 23$

\section{Introduction}

The last three decades have seen the emergence of cosmology as 'precision science', moving from order of magnitude estimates, to predictions and measurements at accuracy levels better than $10 \%$. Cosmic microwave background observations and large galaxy surveys have led this advance in the understanding of the origin and evolution of the Universe. Future surveys promise even higher accuracy, at the $1 \%$ level, over a considerably wider dynamic range than probed earlier. In order to fully utilize the wealth of upcoming data and to address burning questions such as the dynamical nature of dark energy (specified by the equation of state parameter $w=p / \rho, p$ being the pressure, and $\rho$ the density), theoretical predictions must attain at least the same level of accuracy as the observations, even higher accuracy being certainly preferable. The highly nonlinear physics at the length scales probed, combined with complicated gas physics and astrophysical feedback processes at these scales, make this endeavor a daunting task.

As a first step towards achieving the final goal, a necessary requirement is to reach the desired accuracy for gravitational interactions alone, down to the relevant nonlinear scales. Tests with exact solutions such as pancake collapse [1] are valuable for this task, but as shown in [2] the results do not easily translate into statements about the accuracy of different simulation algorithms in realistic cosmological simulations. Exactly solvable problems are typically highly symmetric and hence somewhat artificial. Codes optimized for realistic situations can break down in certain tests even if their results appear to converge in physically relevant settings. Therefore, in order to evaluate the robustness of results from simulation codes, a broad suite of convergence and direct code comparison tests must be carried out.

The codes used in this comparison project are all well established, and have been key drivers in obtaining numerous scientific results. They are based on different algorithms and employ different methods for error control. The code developers have already carried out careful convergence tests themselves and verified to their satisfaction that the codes yield reliable results. But because of the multi-scale complexity of the dynamical problem itself, as well as the incompleteness of most convergence tests, it is necessary to do much more. Therefore, the aim here is to focus on comparing results from a suite of different codes for realistic cosmological simulations. In order to avoid uncertainties from statistical sampling, all codes are run with exactly the same initial conditions, and all results are analyzed using the same diagnostic tools.

The application examples considered here are split into two broad classes, high-resolution runs with hierarchical/hybrid solvers, and medium resolution runs with grid-based codes. Although the grid codes could be run at higher resolution, this would add greatly to the computational expense. Nevertheless, as shown below, 
some very interesting lessons can be learnt regarding the behavior of these codes from our tests; conclusions about higher resolution runs can be drawn from these results.

The paper is organized as follows. In section 2, we describe the ten simulation codes used for the comparison study. In section 3, we briefly describe the simulations carried out for this project. Next, we introduce ParaView in section 4, one of the main analysis tools used in this work. We present our results in section 5 and conclude in section 6.

\section{The codes}

The ten codes used in this paper cover a variety of methods and application arenas. Although many of the codes in principle include gravity, hydrodynamics and other physics (including sub-grid models), here, we focus only on their treatment of gravitational dynamics. All codes use the $N$-body technique, albeit with different implementations. These include parallel particle-in-cell (PIC) methods (the PM codes $\mathrm{MC}^{2}$ and PMM, the PM/AMR codes Enzo and FLASH), a hybrid of PIC and direct $N$-body solvers (the $\mathrm{AP}^{3} \mathrm{M}$ code Hydra), tree algorithms (the tree codes PKDGRAV and HOT) and hybrid tree-PM algorithms (GADGET-2, TPM and TreePM).

The $N$-body method is essentially a dynamical Monte Carlo approach to solving many-body evolution problems. Self-consistent equations of motion are solved for a set of tracer particles which represent a sampling of the system phase space distribution function.

In PIC codes, a computational grid is used to increase the efficiency of the self-consistent inter-particle force calculation. To increase dynamic range, local force computations (e.g. $\mathrm{P}^{3} \mathrm{M}$, tree-PM) and AMR are often used. Treecodes are based on the idea that the gravitational potential of a far-away group of particles is accurately given by a low-order multipole expansion. Particles are first arranged in a hierarchical system of groups in a tree structure. Computing the potential at a point turns into a descent through the tree. Treecodes naturally embody an adaptive force resolution scheme without the overhead of a computational grid. TreePM is a hybrid algorithm that combines a long-range force computation using a grid-based technique, with shorterrange force computation handled by a tree algorithm. In the following, we give a brief description of each code used in this comparison study.

\subsection{The grid codes}

2.1.1. $\mathrm{MC}^{2}$. The multi-species mesh-based cosmology code $\left(\mathrm{MC}^{2}\right)$ code suite includes a parallel PM solver for application to large-scale structure formation problems in cosmology. In part, the code descended from parallel space-charge solvers for studying high-current charged-particle beams developed at Los Alamos National Laboratory under a DOE Grand Challenge [3, 4]. $\mathrm{MC}^{2}$ solves the Vlasov-Poisson system of equations for an expanding universe using standard mass deposition and force interpolation methods allowing for periodic or open boundary conditions with second- and fourth-order (global) symplectic time-stepping and a fast Fourier transform (FFT)-based Poisson solver. The results reported in this paper were obtained using cloud-in-cell (CIC) deposition/interpolation. The overall computational scheme has proven to be efficient while satisfying global constraints: relatively large time-steps are possible with exceptional energy conservation being achieved.

2.1.2. PMM. PMM [5] is an improved PM algorithm that combines high mass resolution with moderate spatial resolution while being computationally fast and memory friendly. The current version utilizes a twolevel mesh FFT-based gravity solver where the gravitational forces are separated into long- and short-range components. The long-range force is computed on the root-level, global mesh, much like in a PM code. To obtain higher spatial resolution, the domain is decomposed into cubical regions and the short-range force is computed on a refinement-level, local mesh. This algorithm achieves a spatial resolution of four times better than a standard one-level mesh PM code at the same cost in memory. In [5], PMM is shown to achieve very similar results to that of $\mathrm{MC}^{2}$ when run with the same minimum grid spacing. 
2.1.3. Enzo. Enzo ${ }^{9}$ is a publicly available, extensively tested AMR, grid-based hybrid code (hydro $+N$-body) which was originally written by Greg Bryan, and is now maintained by the Laboratory for Computational Astrophysics at UC San Diego [6-9]. The code was originally designed to do simulations of cosmological structure formation, but has been modified to examine turbulence, galactic star formation, and other topics of interest. Enzo uses the Berger and Colella method of block-structured AMR [10]. It couples an adaptive particle-mesh method for solving the equations of dark matter dynamics [11, 12] with a hydro solver using the piecewise parabolic method (PPM), which has been modified for cold, hypersonic astrophysical flows by the addition of a dual-energy formalism [13, 14]. In addition, the code has physics packages for radiative cooling, a metagalactic ultraviolet background, star formation and feedback, primordial gas chemistry and turbulent driving.

2.1.4. FLASH. FLASH [15] originated as an AMR hydrodynamics code designed to study X-ray bursts, novae, and Type Ia supernovae as part of the DOE ASCI Alliances Program. Block-structured AMR is provided via the PARAMESH library [16]. FLASH uses an oct-tree refinement scheme similar to [17, 18]. Each mesh block contains the same number of zones $\left(16^{3}\right.$ for the runs in this paper), and its neighbors must be at the same level of refinement or one level higher or lower (mesh consistency criterion). Adjacent refinement levels are separated by a factor of two in spatial resolution. The refinement criterion used is based upon logarithmic density thresholds. Numerous extensions to FLASH have been developed, including solvers for thermal conduction, magnetohydrodynamics, radiative cooling, self-gravity and particle dynamics. In particular, FLASH now includes a multigrid solver for self-gravity and an adaptive particle-mesh solver for particle dynamics. Together with the PPM hydrodynamics module, these provide the core of FLASH's cosmological simulation capabilities. FLASH uses a variable time step leapfrog integrator. In addition to other time step limiters, the FLASH particle module requires that particles travel no more than a fraction of a zone during a time step.

\subsection{The tree codes}

2.2.1. HOT. This parallel tree code [19] has been evolving for over a decade on many platforms. The basic algorithm may be divided into several stages (the method of error tolerance is described in [20]). Firstly, particles are domain decomposed into spatial groups. Secondly, a distributed tree data structure is constructed. In the main stage of the algorithm, this tree is traversed independently in each processor, with requests for nonlocal data being generated as needed. A key is assigned to each particle, which is based on the Morton ordering. This maps the points in three-dimensional space to a one-dimensional list, maintaining spatial locality. The domain decomposition is obtained by splitting this list into $N_{\mathrm{p}}$ (number of processors) pieces. An efficient mechanism for latency-hiding in the tree traversal phase of the algorithm is critical. To avoid stalls during nonlocal data access, effectively explicit 'context switching' is done using a software queue to keep track of which computations have been put aside waiting for messages to arrive. This code architecture allows HOT to perform efficiently on parallel machines with fairly high communication latencies [21]. HOT has a global time stepping scheme. The code was among the ones used for the original Santa Barbara Cluster Comparison Project [22] and also supports gas dynamics simulations via a smoothed particle hydrodynamics (SPH) module [23].

2.2.2. PKDGRAV. The central data structure in PKDGRAV [24] is a tree structure which forms the hierarchical representation of the mass distribution. Unlike the more traditional oct-tree which is used in the Barnes-Hut algorithm [25] and is implemented in HOT, PKDGRAV uses a $k-D$ tree, which is a binary tree. The root-cell of this tree represents the entire simulation volume. Other cells represent rectangular subvolumes that contain the mass, center-of-mass, and moments up to hexadecapole order of their enclosed regions. PKDGRAV calculates the gravitational accelerations using the well-known tree-walking procedure of the Barnes-Hut algorithm. Periodic boundary conditions are implemented via the Ewald summation

9 http://lca.ucsd.edu/codes/currentcodes/enzo 
technique [26]. PKDGRAV uses adaptive time stepping. It runs efficiently on very large parallel computers and has produced some of the world's highest resolution simulations of cosmic structures. A hydrodynamics extension called GASOLINE exists.

\subsection{The hybrid codes}

2.3.1. Hydra. HYDRA [27] is an adaptive $\mathrm{P}^{3} \mathrm{M}\left(\mathrm{AP}^{3} \mathrm{M}\right)$ code with additional $\mathrm{SPH}$ capability. In this paper, we use HYDRA only in the collisionless mode by switching off gas dynamics. The $\mathrm{P}^{3} \mathrm{M}$ method combines mesh force calculations with direct summation of inter-particle forces on scales of two to three grid spacings. In regions of strong clustering, the direct force calculations can become significantly expensive. In $\mathrm{AP}^{3} \mathrm{M}$, this problem is tackled by utilizing multiple levels of subgrids in these high density regions, with direct force computations carried out on two to three spacings of the higher-resolution meshes. Two different boundary conditions are implemented in HYDRA, periodic and isolated. The time-step algorithm in the dark matteronly mode is equivalent to a leapfrog algorithm.

2.3.2. GADGET-2. The $N$-body/SPH code GADGET-2 [28, 29] employs a tree method [25], to calculate gravitational forces. Optionally, the code uses a tree-PM algorithm based on an explicit split in Fourier space between long- and short-range forces [30]. This combination provides high performance while still retaining the full spatial adaptivity of the tree algorithm, allowing the code to reach high spatial resolution throughout a large volume. By default, GADGET-2 expands the tree multipoles only to monopole order, in favor of a compact tree storage, a cache-optimized tree-walk, and consistent and efficient dynamic tree updates. The cell-opening criterion used in the tree walk is based on an estimator for the relative force error introduced by a given particle-cell interaction, such that the tree force is accurate up to a prescribed maximum relative force error. The latter can be lowered arbitrarily, if desired, at the expense of higher calculation times. The PM part of GADGET-2 solves Poisson's equation on a mesh with standard FFTs, based on a CIC mass assignment and a four-point finite differencing scheme to compute the gravitational forces from the potential. The smoothing effects of grid assignment and interpolation are corrected by an appropriate deconvolution in Fourier space. The time-stepping of GADGET-2 uses a leap-frog integrator which is symplectic in case constant timesteps (in the log of the expansion factor) are employed for all particles. However, the code is normally run in a mode where individual and adaptive timesteps are used to speed up the calculation time. To this end, the timesteps for the short-range dynamics are allowed to freely adapt to any power of two subdivision of the long-range timestep. GADGET-2 is fully parallelized for massively parallel computers with distributed memory, based on the MPI standard. The code can also be used to simulate hydrodynamical processes using the particle-based SPH method (e.g. [31]), in an entropy conserving formulation [32], a feature which is however not exercised in the simulations considered in this paper.

2.3.3. TPM. TPM $[33,34]$ is a publicly available hybrid code combining a PM and a tree algorithm. The density field is broken down into many isolated high-density regions using a density threshold criterion. These contain most of the mass in the simulation but only a small fraction of the volume. In these regions, the gravitational forces are computed with the tree algorithm while for the bulk of the volume the forces are calculated via a PM algorithm, the PM time steps being large compared to the time-steps for the tree-algorithm. The PM algorithm uses the CIC deposition/interpolation scheme and solves the Poisson equation using FFTs. The time integrator in TPM is a standard leap-frog scheme: the PM time steps are fixed whereas tree particles have individual time steps, half of the PM step or smaller.

2.3.4. TreePM. The algorithmic structure of the Tree-PM code [35] is very similar to GADGET-2. The particles are integrated using a second-order leap-frog method, with position and canonical momentum as the variables. The time step is dynamically chosen as a small fraction (depending on the smoothing length) of the local free-fall time and particles have individual time steps. The force on any given particle is computed in two stages. The long-range component of the force is computed using the PM method, while the shortrange component is computed from a global tree. A spline softened force law is used. The tree expands forces 
to monopole order only, and cells are opened based upon the more conservative of a geometric and relative force error criterion. The PM force is computed by direct FFT of the density grid obtained from CIC mass assignment.

\section{The simulations}

A previous code comparison suite [2] considered three cosmological test problems: the Santa Barbara cluster [22], and two large-scale structure simulations of $\Lambda \mathrm{CDM}$ models in a $64 h^{-1} \mathrm{Mpc}$ box and a $256 h^{-1} \mathrm{Mpc}$ box. In the latter two cases, the primary target of this previous work was to investigate results in a medium resolution regime, addressing statistical quantities such as the two-point correlation function, the density fluctuation power spectrum, and the dark matter halo mass function.

In this paper, we focus further attention on one of these tests, the smaller of the $\Lambda$ CDM boxes. Due to the small box size, the force resolution of all codes - including the pure mesh codes - is, in principle, sufficient to analyze properties of individual halos themselves. This allows us to extend the dynamic range of the code comparison to higher resolution than studied earlier. In this new regime, we expect to see a much broader divergence of results because of the more demanding nature of the test. (Even in the previous analysis [2], the power spectrum was unexpectedly deviant at the larger wavenumbers considered.) Our aim is to characterize the discrepancies and attempt to understand the underlying causes.

All codes were given exactly the same particle initial conditions at a redshift $z_{\text {in }}=50$. The initial linear power spectrum was generated using a fit to the transfer function [36], a modification of the BBKS fit [37]. This fit does not capture baryon oscillations but takes baryonic suppression into account (these details are of only limited relevance for the test). The cosmology underlying the simulations is given by $\Omega_{\mathrm{CDM}}=0.27$, $\Omega_{\mathrm{b}}=0.044, \Omega_{\Lambda}=0.686, h=0.71, \sigma_{8}=0.84$, and $n=0.99$. The simulation was run with $256^{3}$ particles, which leads to an individual particle mass of $m_{\mathrm{p}}=1.362 \times 10^{9} h^{-1} M_{\odot}$.

While performing a comprehensive code comparison study which involves very different algorithms - such as grid and particle-based methods in the present case- a central and difficult question immediately arises: what is the most informative way to compare the codes and learn from the results? The difficulty is compounded by the fact that codes are often optimized under different criteria and controlling numerical error is a complex multi-parameter problem in any case, even for codes that share the same general underlying algorithm.

As a case in point, let us consider the choice of force resolution for each code. (Since the volume and number of particles are fixed, the mass resolution is the same for each run.) One option would be to run all codes with the same formal force resolution. But practical limitations of the pure PM codes would then impose an unrealistically low force resolution on the others. (In addition, one also has the problem that it is not easy to compare resolutions across different algorithms; moreover, time-stepping errors also must be folded into these sorts of estimates.) Such a comparison would be rather uninteresting, because realistic cosmological simulations are run with higher resolutions than would be possible in a conservative test of this type: interesting effects on small scales would be missed.

A more uncontrolled, but nevertheless useful option is to allow every simulator to run her or his code with close to the optimal settings they would also use for a scientific run (given the other restrictions imposed by the test problem). In this case, a more useful comparison can be performed in which we can access the robustness of conclusions from cosmological simulations, taking into account the various characteristics of the individual codes, including the scales down to which their results are expected to be valid. Here, while our approach adheres more closely to the second strategy, we do try to assess at what length scales one should expect a specific code to break down assuming that the resolution of the code is accurately estimated by the simulator. Our analysis is not aimed at completeness but at tracking down discrepancies due to both algorithmic or resolution-induced effects.

The nominal resolutions for the different codes for the performed runs are as given in table 1 . We have converted the different softening kernels into Plummer equivalents following the normalization conventions of [38]. We have matched the different softening kernels $\phi$ at zero and compared them at this point. With the 
Table 1. Softening lengths measured in $h^{-1} \mathrm{kpc}$. The different smoothing kernels have been converted into Plummer softening equivalents by matching the potential at the origin. While this procedure is only approximate, it makes a comparison of the different force resolutions more meaningful. For details on the conversion see the main text. The two AMR codes Enzo and FLASH were run at a root grid resolution of $250 h^{-1} \mathrm{kpc}$.

\begin{tabular}{cccccccccc}
\hline MC $^{2}$ & PMM & Enzo & FLASH & HOT & PKDGRAV & Hydra & GADGET-2 & TPM & TreePM \\
\hline 62.5 & 62.5 & 62.5 & 62.5 & 7.1 & 1.6 & 28.4 & 7.1 & 5.1 & 5.7 \\
\hline
\end{tabular}

normalization conventions in [38], we find:

$$
\begin{aligned}
& \phi_{\text {Plummer }}(0) \propto \frac{1}{\epsilon}, \\
& \phi_{\text {Spline }}(0) \propto \frac{7}{5} \frac{1}{\epsilon}, \\
& \phi_{\mathrm{K}_{3}}(0) \propto \frac{2079}{512} \frac{1}{\epsilon},
\end{aligned}
$$

where $\epsilon$ is the softening length. The grid resolution of the PM and AMR codes is roughly equivalent to the Plummer softening. HOT and Hydra have Plummer force kernels implemented, PKDGRAV uses Dehnen's $\mathrm{K}_{3}$ kernel [38] and the three tree-PM codes use spline kernels. With the above definitions, it is easy to convert the spline and $\mathrm{K}_{3}$ kernels into Plummer via

$$
\begin{aligned}
\epsilon_{\text {Spline }} & =1.4 \epsilon_{\text {Plummer }}, \\
\epsilon_{\mathrm{K}_{3}} & =4.06 \epsilon_{\text {Plummer }},
\end{aligned}
$$

which we used to standardize the force resolution quotes in table 1. We note that some of the codes below could have been run at higher resolution, and the values below should not be thought of as resolution limits. In fact, the choices of these values represent compromises due to run time considerations as well as a (loosely) pre-planned scatter to try and determine the effects of force resolution on the simulation results.

\section{Analysis framework and tools}

Broadly speaking, the major aim of our code comparison project is to characterize differences in results from large cosmological simulation codes, identify the causes underlying these differences, and, if possible, develop strategies to reduce or eliminate the differences in order to obtain reliable results over large length and mass scales. If it is not possible to eliminate some of the differences, e.g. due to insufficient force resolution in grid codes, it is still important to provide robust criteria that correctly determine the scales at which the code can be trusted, and at what level.

The identification and characterization of differences in code results is not as straightforward as it first appears. Certainly we may, and do, compare standard statistical quantities such as low-order correlation functions. However, there is much more information in the data beyond this, e.g. large-scale structure morphology, substructure in the density field, and subtle features such as the variation in halo bias as a function of the local environment. For these reasons, it is often very useful to simply look at the region or object of interest in the simulation and compare it across the different codes. Following this qualitative comparisonperhaps even inspired by it - the aim is to construct a hypothesis about the cause for the perceived difference which then has to be carefully tested with quantitative measures.

It is very desirable to have a framework which combines these two steps in a convenient, and eventually seamless, manner. The framework should allow differences and anomalies picked up by eye from data sets to be immediately queried and quantified using a programmable toolkit. An example relevant to cosmological 


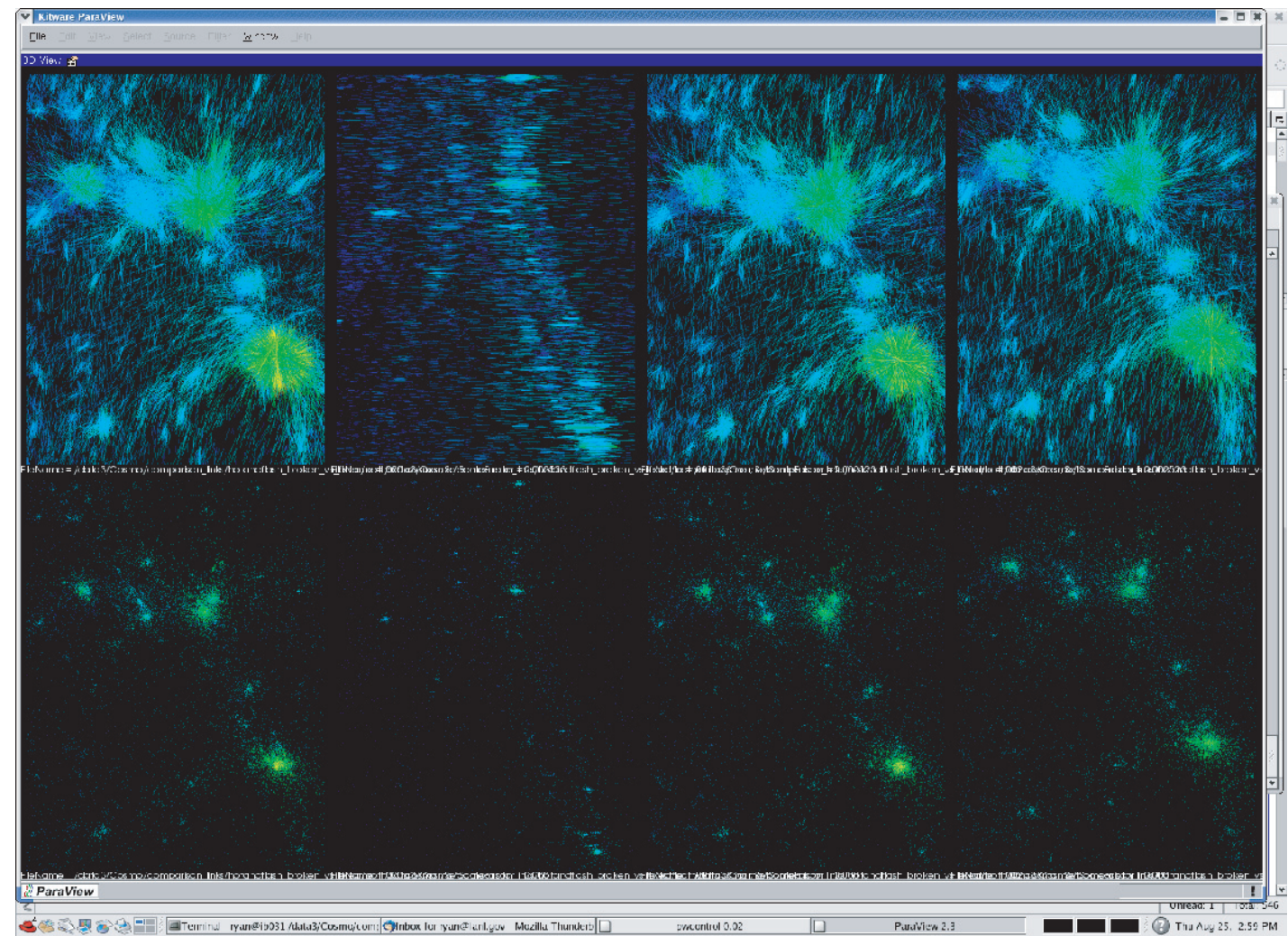

Figure 1. Screenshot of the comparative visualization manager in ParaView. Upper row: results from four different codes, zoomed into a dense region of the simulations. Particles are displayed as arrow glyphs, colored with respect to their velocity magnitude. Lower row: same region, the particles now displayed simply as dots.

simulations is the following. Suppose we visually identify fewer halos in one simulation compared to another. The analysis tool should then provide quantitative information of the following type: in what areas is the difference larger, what is the exact number count of the halos in this region, what is the difference in the environment (e.g. by comparing the local density in the two codes), what is the halo history in the region, and so on.

As part of this paper, we include an introduction of ParaView ${ }^{10}$ [39] to the cosmology and the wider computational communities. ParaView has some of the features discussed above built in and allows the user to implement additional analysis tools. ParaView is an open-source, scalable visualization tool which is designed as a layered architecture. The foundation and first layer of ParaView is the visualization toolkit (VTK). VTK provides data representations, algorithms, and a mechanism to interconnect these to form a working program. The second layer is a parallel extension to the VTK which supports streaming of all data types and parallel execution on shared and distributed memory machines. The third layer is ParaView itself. ParaView provides a graphical user interface and transparently supports the visualization and rendering of large datasets via hardware acceleration, parallelism and level-of-detail techniques.

For the code comparison project, we have implemented a particle reader which works with a simple data format; the format has binary information about particle positions, velocities, masses and particle tags. This format was used by all the codes in the tests. This allows other simulators who wish to test their codes against our results to use exactly the same analysis tool. As explained later, we have also implemented a diverse set of diagnostic tools relevant for cosmological simulations. These help to ease the analysis of large simulation data sets and make it more efficient. We plan to extend the set of available analysis features in the near future.

${ }^{10}$ We use ParaView 2.6 throughout this paper. This is the latest stable release which can be downloaded at http://www.paraview. org/HTML/Download.html. 

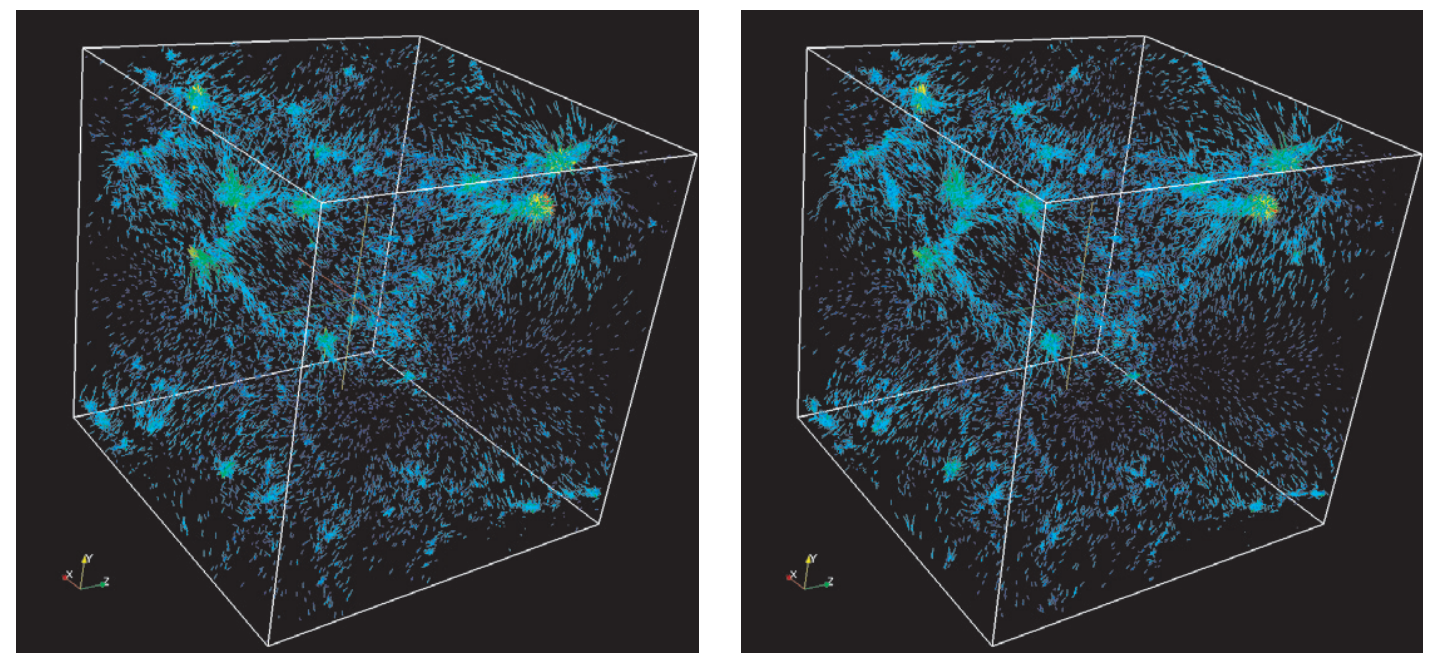

Figure 2. A subset of 20000 particles at $z=0$ from the GADGET-2 simulation (left) and the Enzo simulation (right). The particles are shown with vector arrow glyphs which are sized and colored by their velocity magnitude (blue: slowest, red: fastest).

\section{Results}

\subsection{Results for the full simulation box}

As an initial test, a simple view of the simulation output at $z=0$ proves to be very useful. ParaView offers a comparative visualization option in which the results from different simulations can be shown simultaneously. Manipulation on any one output in this mode results in the same manipulation for all the others. ParaView allows fly-ins, rotation of the box, projections, and has many more features which make it convenient to inspect the outcome of the simulation. A screenshot of the comparative visualization manager is displayed in figure $1-$ a zoom into an arbitrary region of the simulation box showing simultaneous results from four different codes. In the upper row, a subset of the particles is shown as arrow glyphs, colored by velocity magnitude, the lower row shows the particles as dots with the same coloring scheme. A quick inspection of these snapshots reveals that the code 2 run had a problem with the velocities and code 4 had slightly incorrect boundary conditions (the whole picture being shifted upward). (Of course these initial bugs were fixed before going on to the final results discussed below!)

Figure 2 shows a comparison of the final GADGET-2 and Enzo outputs. We show a subsample of 20000 particles, each displayed with vector arrow glyphs, sized and colored by their velocity magnitude. The arrow glyphs nicely represent the flows in the box to the major mass concentrations. As to be expected, particles in the field are slow (blue), while the particles in the halos have the largest velocities (yellow to red). While the overall appearance of both simulations shown is very similar, subtle differences can be seen (e.g. there are no small structures in the flow regions in the Enzo simulations), indicating the higher resolution employed in the GADGET-2 run. (Five of the biggest halos in the simulation will be examined in more detail below, the resolution differences becoming significantly more apparent.)

\subsection{Dark matter halos}

The halo paradigm is central to any large-scale structure analysis; dark matter in simulations, discretized in the form of heavy, collisionless particles, forms clearly visible filaments (stripes) and halos (clumps of dark matter) through the process of gravitational instability. Figure 2 shows these structures clearly for the simulations studied in this paper. The halo paradigm agrees well with observations of galaxy rotation curves, and velocity dispersions of galaxies in clusters which favor scenarios where luminous, baryonic matter is embedded in massive, extended, and close to spherical conglomerates of dark matter. In simulations, dark matter halos can be identified and 'weighed' in different ways. We can measure overdensities (see e.g. [40]) 
Table 2. Halo 3 data: distance of the center from the mean value for all codes, and the mass of the halo from different simulations.

\begin{tabular}{lrrrc}
\hline Code & $\Delta X_{c}\left(h^{-1} \mathrm{kpc}\right)$ & $\Delta Y_{c}\left(h^{-1} \mathrm{kpc}\right)$ & $\Delta Z_{c}\left(h^{-1} \mathrm{kpc}\right)$ & Mass $\left(10^{14} h^{-1} M_{\odot}\right)$ \\
\hline MC $^{2}$ & -86.23 & 158.81 & -14.68 & 2.749 \\
PMM & 201.68 & 33.90 & 10.24 & 2.757 \\
Enzo & -21.36 & 45.16 & 11.36 & 2.745 \\
FLASH & -41.66 & -22.56 & -23.10 & 2.726 \\
HOT & -30.02 & -120.54 & 43.99 & 2.720 \\
PKDGRAV & 38.58 & 52.19 & -43.98 & 2.679 \\
Hydra & 19.91 & -28.29 & 0.77 & 2.721 \\
GADGET-2 & -27.08 & -59.00 & -0.70 & 2.705 \\
TPM & -36.37 & -35.09 & 1.04 & 2.697 \\
TreePM & -17.45 & -24.62 & 13.63 & 2.727 \\
\hline
\end{tabular}

or use group finding algorithms such as friends-of-friends (FOF) algorithms [41] to find halos. For a recent discussion of the different halo finding algorithms see, e.g., $[44,45]$.

A remarkable feature of halos was found by Navarro, Frenk, and White (hereafter NFW) [42]: dark matter halos of all masses, from dwarf galaxies to the largest clusters of galaxies, have spherically averaged density profiles that are well described by a single 'universal' formula

$$
\rho(r)=\frac{\rho_{\mathrm{c}} \delta_{\mathrm{c}}}{r / r_{\mathrm{s}}\left(1+r / r_{\mathrm{s}}\right)^{2}} .
$$

The scaling radius, $r_{\mathrm{s}}$, and characteristic overdensity, $\delta_{\mathrm{c}}$, are free parameters of the model, while $\rho_{\mathrm{c}}$ is the critical density for closure of the Universe. Even though a theoretical explanation for this universal profile has not been found, the nature of the profile itself has received extensive support from simulations carried out by many different groups [43] (there do remain questions about the behavior at very small radii, but these are not relevant here).

Here, we are interested in the variation of the profiles produced by the different codes, tending towards the outer region of the halo. This variation may be significant for determining halo masses via the often used FOF algorithm. The mass that the halo finder will 'see', strongly depends on the density and density gradient close to the virial radius $\left(R_{200}\right)$ of a halo. On the other hand, capturing the inner slope of a halo profile is the prime test of the code's force resolution. On scales below this resolution limit, particle positions get randomized, resulting in a flattened density profile (numerical errors can also lead to a sharpening of the profile due to an associated unphysical damping).

We first compare the five heaviest halos from the simulations; their masses range between approximately $2-5 \times 10^{14} h^{-1} M_{\odot}$, thus each halo is sampled with 150000 or more particles. The individual halo masses (as found by the FOF algorithm) are in agreement within 3\% for all ten codes. Note that the FOF masses found for the grid codes are slightly higher. This is presumably due to their lower resolution in this comparison, resulting in less tight halos. The FOF halo finder can identify more particles in the fuzzier outskirts of lower resolution simulations as belonging to the halo than in the high resolution runs. The centers of the halos are defined by the minimum of the local potential of the halo. (The halo centers were found by applying a single, uniform method across all the code results.) Here, the agreement among the codes is even better than for the masses - the difference is less than $0.5 \%$ of the box size. In table 2 , we show the center and mass of one of the halos, halo 3. This halo (also shown in figure 7) has the size and mass of a group of galaxies. The dispersion in the mass and position of the center is similar for the other halos, whose profiles we investigate next.

In figure 3, we present the spherically averaged density profiles for the five heaviest halos in the simulation. As an arbitrary reference, the black line represents the best NFW fit (equation 6) for the TPM data. The fit is shown up to the inner $10 h^{-1} \mathrm{kpc}$ of each halo. In addition, we show two residual panels for each halo profile. The upper panel shows the ratio of all codes with respect to GADGET-2, while the lower panel shows only the four grid codes and ratios with respect to $\mathrm{MC}^{2}$. 

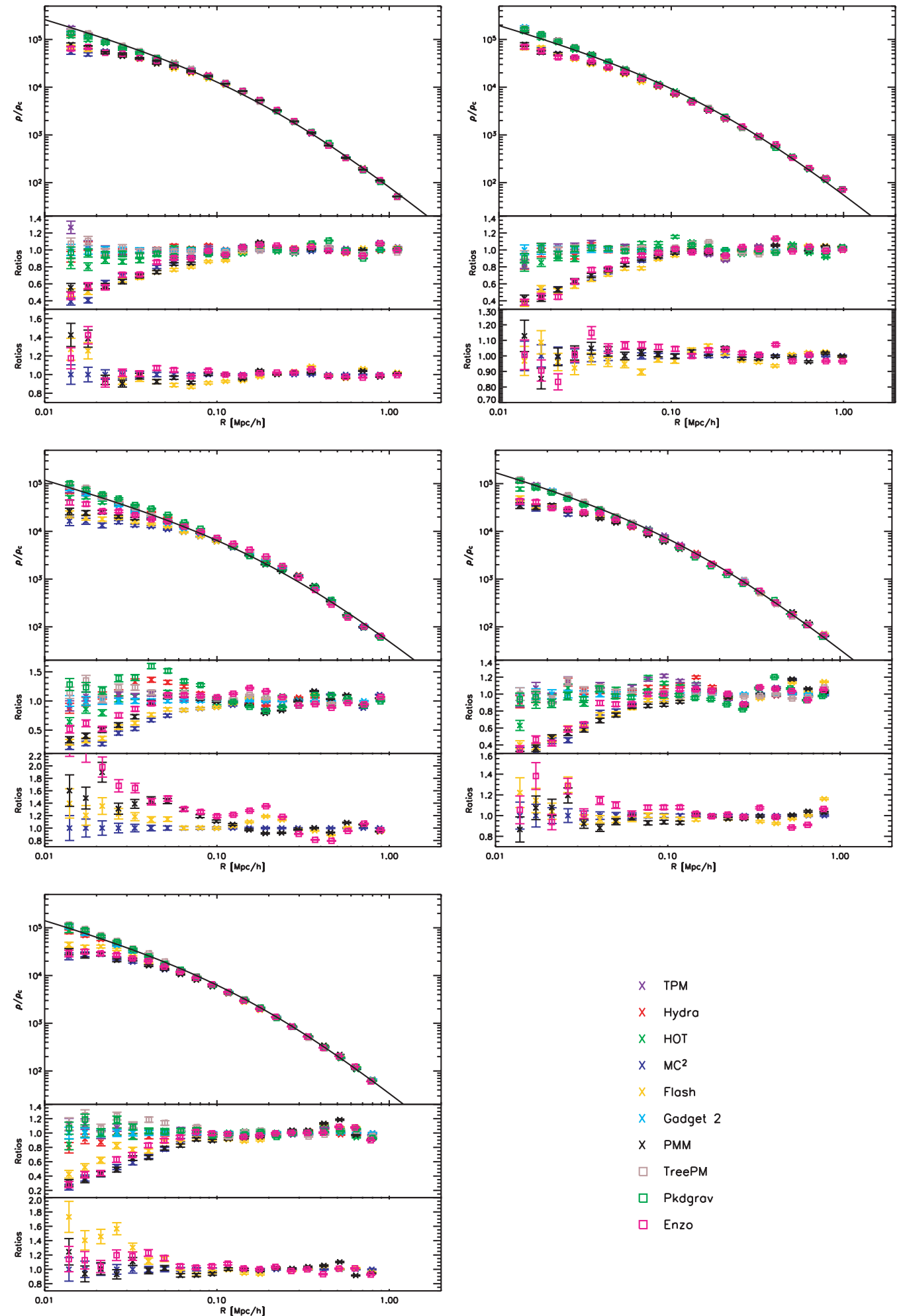

$$
\begin{array}{ll}
\times & \text { TPM } \\
\times & \text { Hydra } \\
\times & \text { HOT } \\
\times & \text { MC2 } \\
\times & \text { Flosh } \\
\times & \text { Gadget } 2 \\
\times & \text { PMM } \\
\square & \text { TreePM } \\
\square & \text { Pkdgrov } \\
\square & \text { Enzo }
\end{array}
$$

Figure 3. Halo profiles for the five heaviest halos in the simulation (heaviest, upper left corner). The last data point on the profiles corresponds to the halo's virial radius $\left(R_{200}\right)$. The black line shows the best-fit NFW profile to the TPM simulation, mainly to guide the eye. In the outer regions all codes agree very well. In the inner regions the fall-off of the grid codes is as expected due to resolution limitations. The fall-off point can be predicted from the finite force resolution and agrees well with the results. The middle panel in each plot shows the ratio of the different codes with respect to GADGET-2. The lower panels show only the four grid codes and the ratio with respect to $\mathrm{MC}^{2}$. 

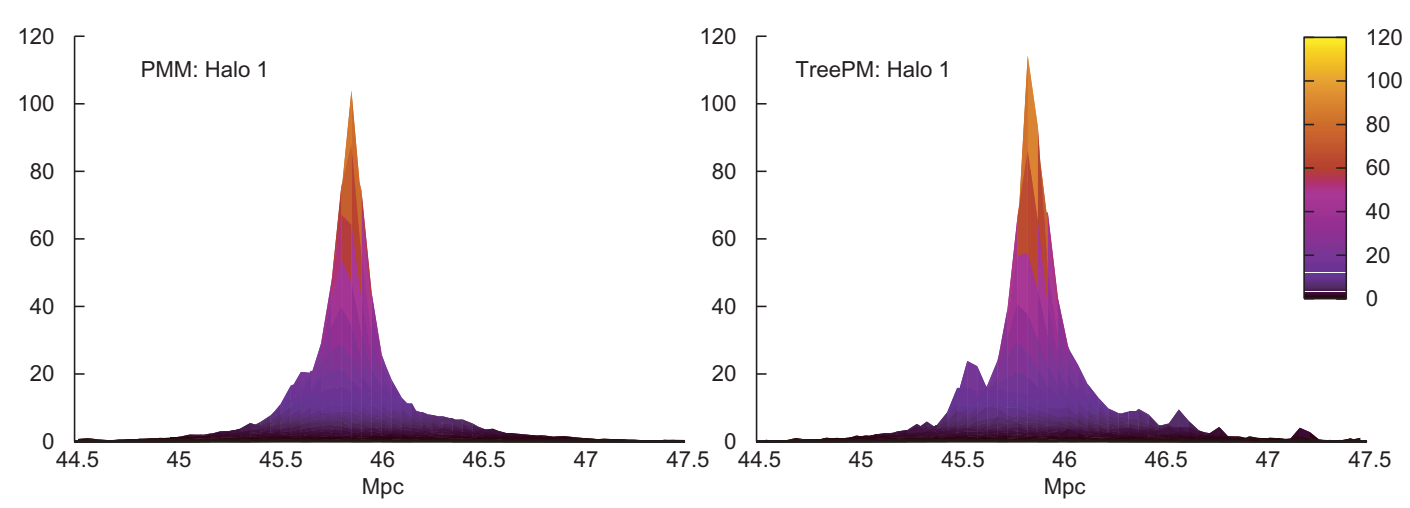

Figure 4. Projected and normalized two-dimensional density for halo 1 from PMM (left) and TreePM (right) viewed along the $y$-axis. All the particles within a sphere of radius $2 R_{200}$ have been projected on a $100 \times 100$ grid in $x$ and $y$. The local density has been divided by the mean density in that region, which is very close for all codes. TreePM has a slightly higher density in the inner region of the halo than PMM, as to be expected from the different force resolutions. Overall, the agreement is very good.

The agreement in the outer part of the halos is excellent. As expected, the codes exhibit different behaviors on small scales (depending on their force resolution and time-stepping), thus the inner parts of halos are not always the same. While the high resolution codes successfully track the profile all the way into the plotting limits of figure 3, the profiles from the mesh codes depart much earlier $\left(60-100 h^{-1} \mathrm{kpc}\right)$, with approximately constant density in the core. The onset of the flattening is consistent with the nominal resolution of the grid codes, which is given in table. 1. Note that among the mesh codes there is no significant difference between the fixed mesh codes which ran at the highest resolution throughout the whole simulation volume, and the AMR codes whose base mesh spacing is a factor of four times lower.

We now study three of the five halos in more detail, restricting attention to particles within a sphere of radius $2 R_{200}$. The profiles of the largest halo, halo 1 , shown in figure 3 , agree well down to $R=0.06 h^{-1} \mathrm{Mpc}$; at smaller scales the finite resolution of the grid codes becomes apparent. Nevertheless, the grid codes and the high-resolution codes among themselves yield very consistent results. Figure 4 shows the density of halo 1 for the lower resolution code PMM and the higher resolution code TreePM in two-dimensional projection. The two-dimensional density field is computed on a $100 \times 100$ grid within the $2 R_{200}$ region, projected onto the $z$-direction (another projection along the $x$-direction is also shown). The projected density field has been normalized by dividing out the mean density in this area. The mean density is very close across the different codes, hence the normalization allows for direct comparisons of the projected density fields. As mentioned earlier, the positions of the halo centers (density peaks) are in remarkably good agreement. Due to its higher resolution, the density in the center of the halo from the TreePM run is slightly higher (as to be expected from the profiles). In addition, TreePM shows slightly more substructure on the outskirts of the halo, displayed by the small 'hills'. Overall, the halo is very smooth and well defined, which is reflected in the good agreement of the profiles. The density plots for the four grid codes are very similar. The small structures around the halo in the other codes also show only very minor variations, thus the PMM and TreePM results can be considered to be representative.

The profiles of halo 3 show substantially more variation among the different codes in the inner region, relative to the other four halos. Studying it in more detail, we first investigate a subset of four codes: $\mathrm{MC}^{2}$ FLASH, GADGET-2 and HOT, covering a wide range of force resolutions. In figure 5, we show a zoom into the center of the halo. The particles are shown in white. Superimposed on the particle distribution is a twodimensional density contour evaluated on a $100 \times 100$ grid and smoothed with a Gaussian filter, projected along the $z$-direction. The contouring and filtering are intrinsic functions in ParaView. ParaView provides a Gaussian filter called vtkGaussianSplatter. This is a filter that injects input points into a structured points (volume) dataset. As each point is injected, it 'splats' or distributes values to nearby voxels. Data are distributed using an elliptical, Gaussian distribution function. The distribution function is modified using scalar values (expands distribution) or normals (creates ellipsoidal distribution rather than spherical). 

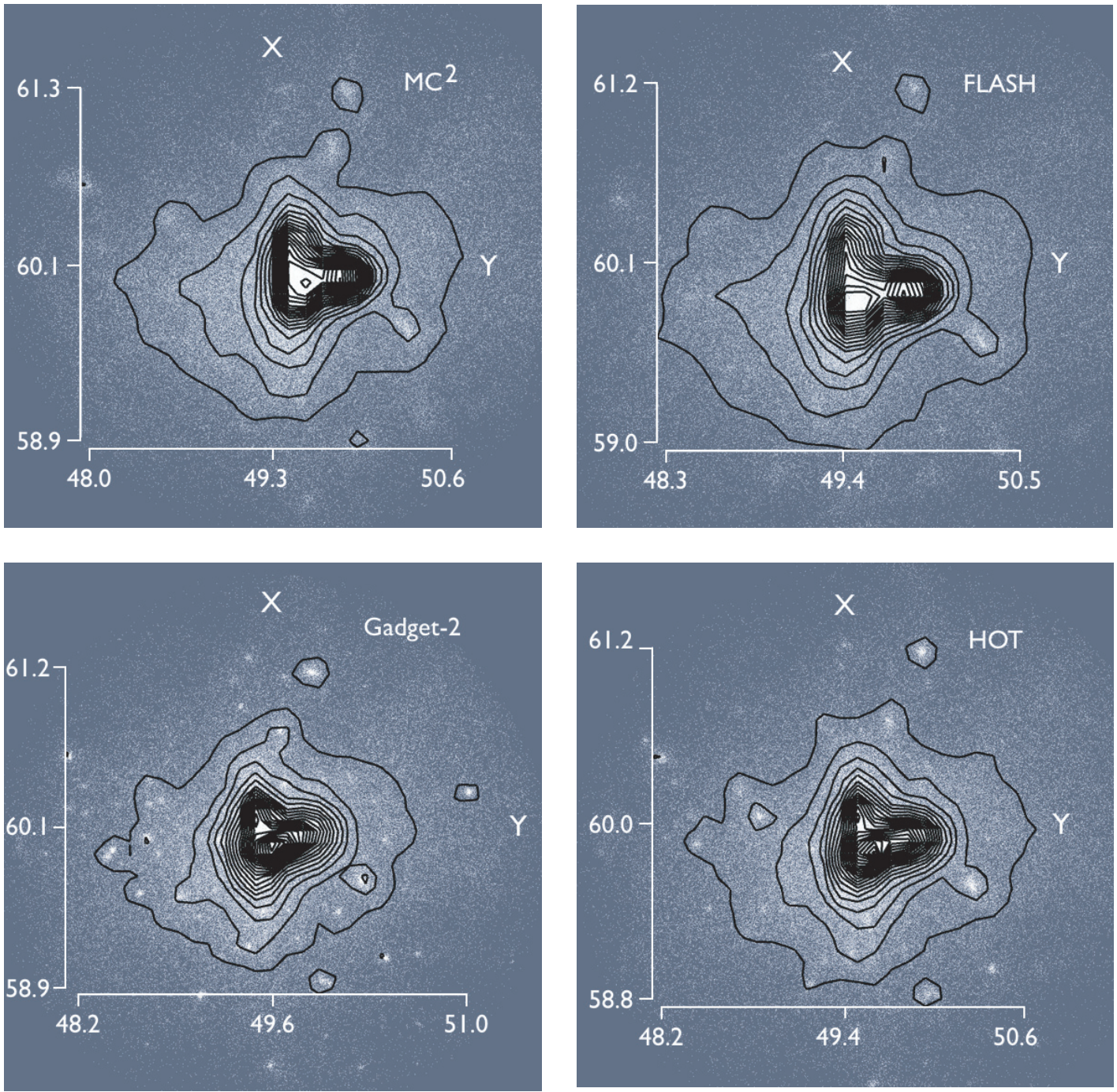

Figure 5. Two-dimensional contour plot of the projected density for halo 3 from $\mathrm{MC}^{2}$, FLASH, GADGET-2, and HOT (left upper to right lower plot). White: particles, black: contour smoothed with a Gaussian filter.

The overall appearance of the halo is remarkably similar between the codes, a major feature of the halo being its irregular shape. The left side of the halo is elongated and a second major peak has developed on the right, leading to a triangular shape in this projection. This irregularity (seen also very clearly in figure 6) is most likely the reason for the disagreement in the inner part of the profiles. The halo has probably undergone a recent merger or is in the process of merging. Comparing the lower resolution runs from $\mathrm{MC}^{2}$ and FLASH with GADGET-2 and HOT, the effect of force resolution is very apparent, the high resolution runs producing significantly more substructure. GADGET-2 shows slightly more substructure than HOT, which could be due to the adaptive time stepping used in the GADGET-2 run relative to HOT's global time-step.

Figure 7 shows halo 3 from the remaining six runs. As in figure 4, the two-dimensional density is shown on a $100 \times 100$ grid. The three-dimensional view underlines the rather complicated structure of the halo. PMM and Enzo show the elongated structure with two maxima, whereas the Hydra and PKDGRAV results differ somewhat from the other codes. They have a more well-defined peak and do not exhibit much of the second structure. TreePM and TPM are very similar to GADGET-2 and HOT. Overall, halo 3 has much more interesting features than halo 1, which leads to slight discrepancies in the halo profiles among the codes.

Lastly, we study halo 4 from a subset of the codes: $\mathrm{MC}^{2}$, GADGET-2, PKDGRAV and HOT, covering the grid, treePM and tree codes. The results are shown in figure 8 . As before, the lower density of the PM code is 

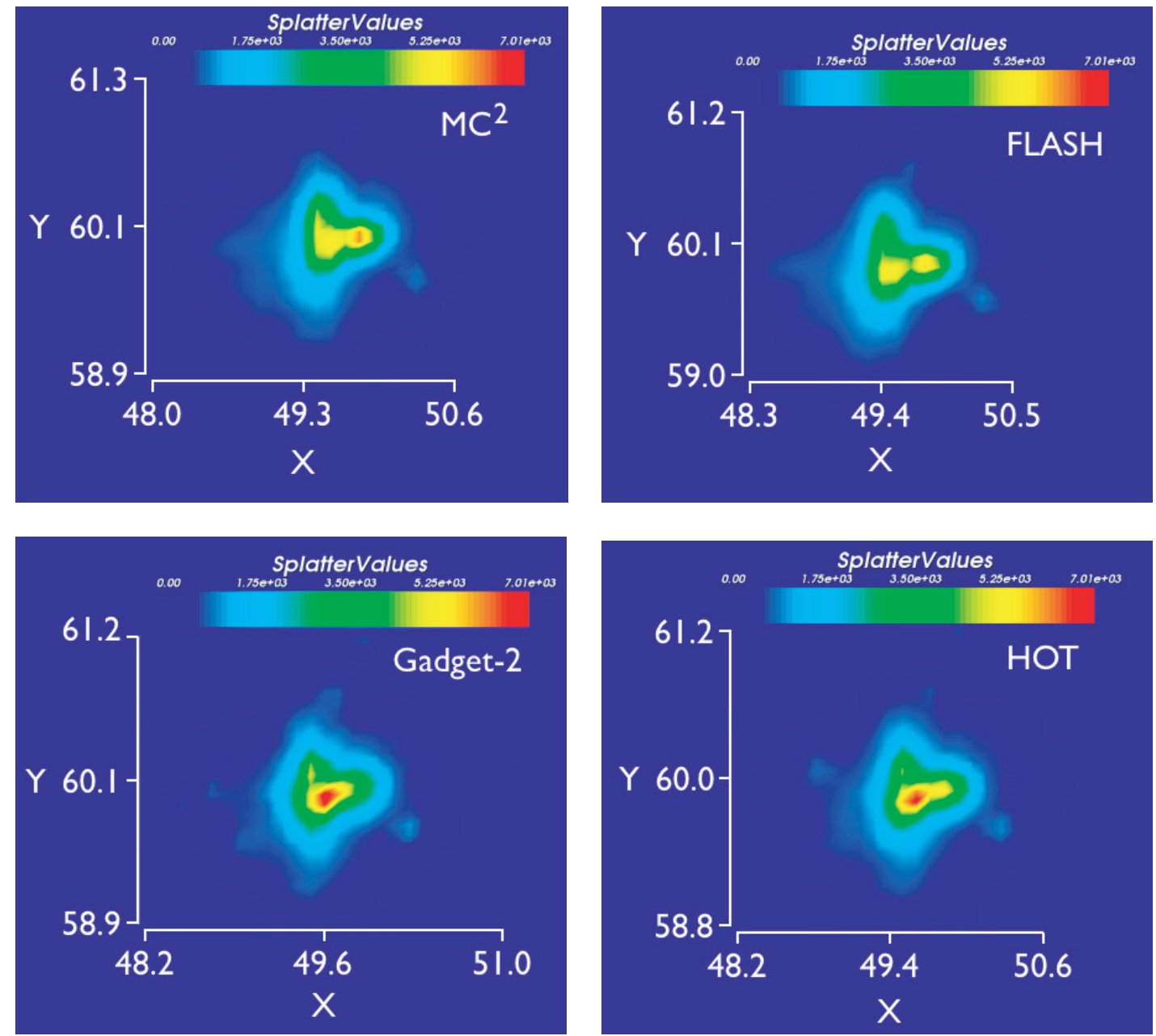

Figure 6. Same halo as in figure 5 for the same codes: $\mathrm{MC}^{2}$, FLASH, GADGET-2, and HOT. This time the density itself is shown.

due to its restricted resolution. Overall, the agreement is again very satisfying. The centers of the halos are in excellent agreement, and all four runs show a smaller structure on the left of the main halo. The exact details of the smallest structures are different which could be due to inaccurate time-stepping and discrepancies in the codes' output redshifts.

Overall, the comparison of the largest halos in the box is very satisfactory. The halo profiles agree on the scales expected from the code resolutions. Differences of the inner parts can be explained due to very irregular shapes as in halo 3. The reader should keep in mind that we did not resimulate the halos with higher resolution, and that these halos were extracted straight out of a cosmological volume simulation. Therefore, the level of agreement is in accord with theoretical expectations.

\subsection{The mass function and halo counts as a function of density}

5.3.1. The mass function. An important statistic in cosmology is the number count of halos as a function of mass, the so-called mass function. The mass function of clusters of galaxies from ongoing and upcoming surveys can provide strong constraints on dark energy [46]. Numerous studies have been carried out to predict the mass function theoretically [47-49]. Because halo formation is a strongly nonlinear dynamical process, the approximations underlying analytic predictions limit the attainable accuracy for constraining cosmological parameters. Nevertheless, some of these analyses can help to understand the origin and qualitative behavior of the mass function. 

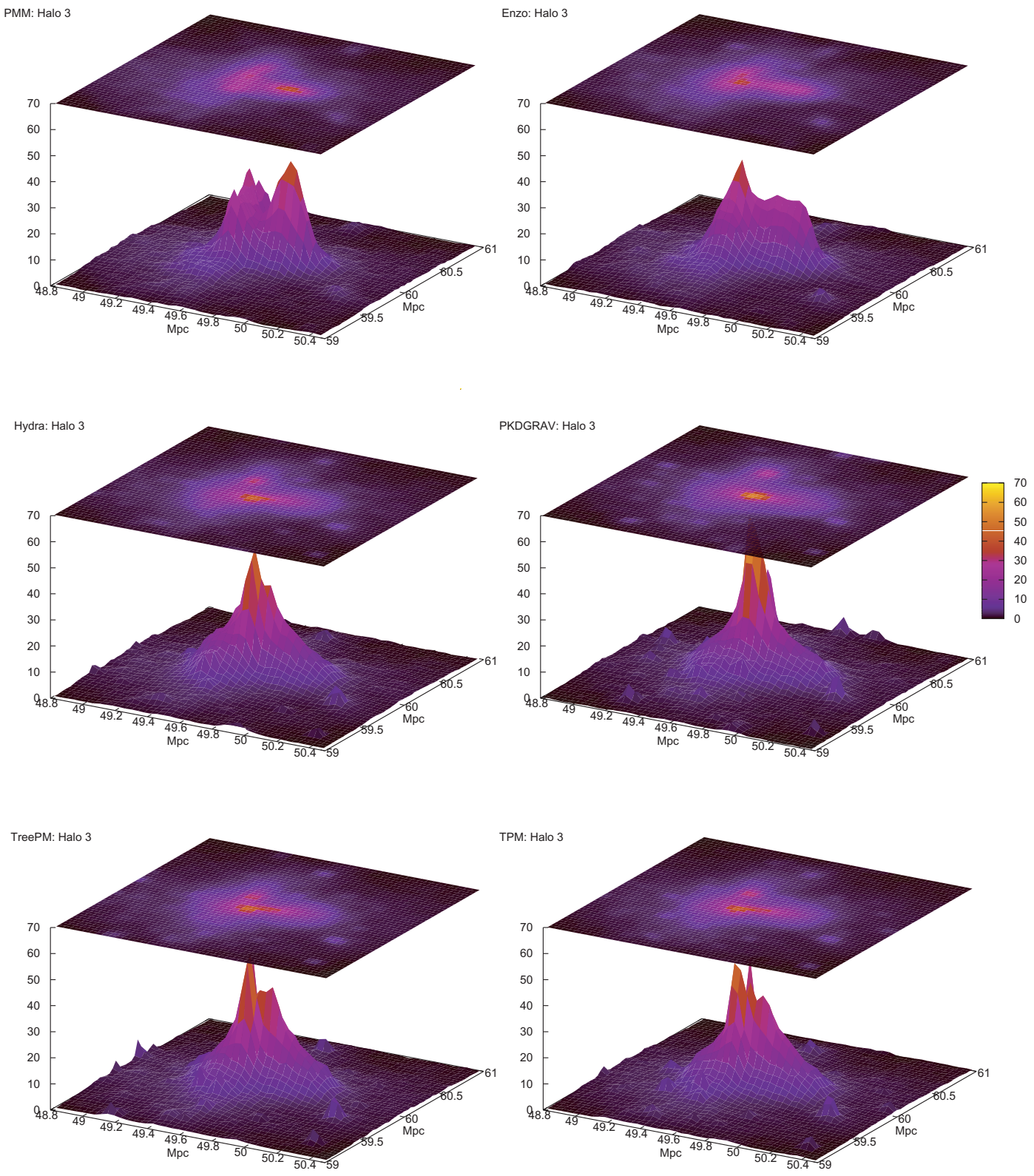

Figure 7. Projected two-dimensional densities from PMM, Enzo, Hydra, PKDGRAV, TreePM and TPM for halo 3. The two-dimensional density field was obtained in the same way as in figure 4 . The $x$ - and $y$-axes extend to $2 R_{200}$ for each halo. The panel on the top of each graph shows the projected density. The color coding is the same for each plot, shown in the result for PKDGRAV.

In order to obtain more precise predictions, several groups have carried out large $N$-body simulations to find better fits for the mass function [50-54]. In addition, the evolution of the mass function has been studied in detail [53-56]. The numerical study of the mass function poses several challenges to the simulation code, especially if one wants to obtain reliable results at the few per cent level: the number of particles in a halo has to be sufficient in order to prevent systematic biases in determinations of the halo mass [52], the force resolution has to be adequate to capture the halos of interest $[55,56]$, the simulation has to be started at sufficiently high redshift $[55,56]$, and finite box corrections might have to be considered if the simulation box is small [56-58] (for a comprehensive study of possible systematic errors in simulating the mass function and its evolution, see [56]).

In this paper, we study the mass function at $z=0$. We identify halos with a FOF algorithm [41] with linking length of $b=0.2$. The smallest halo we consider has 10 particles, not because this is physically 

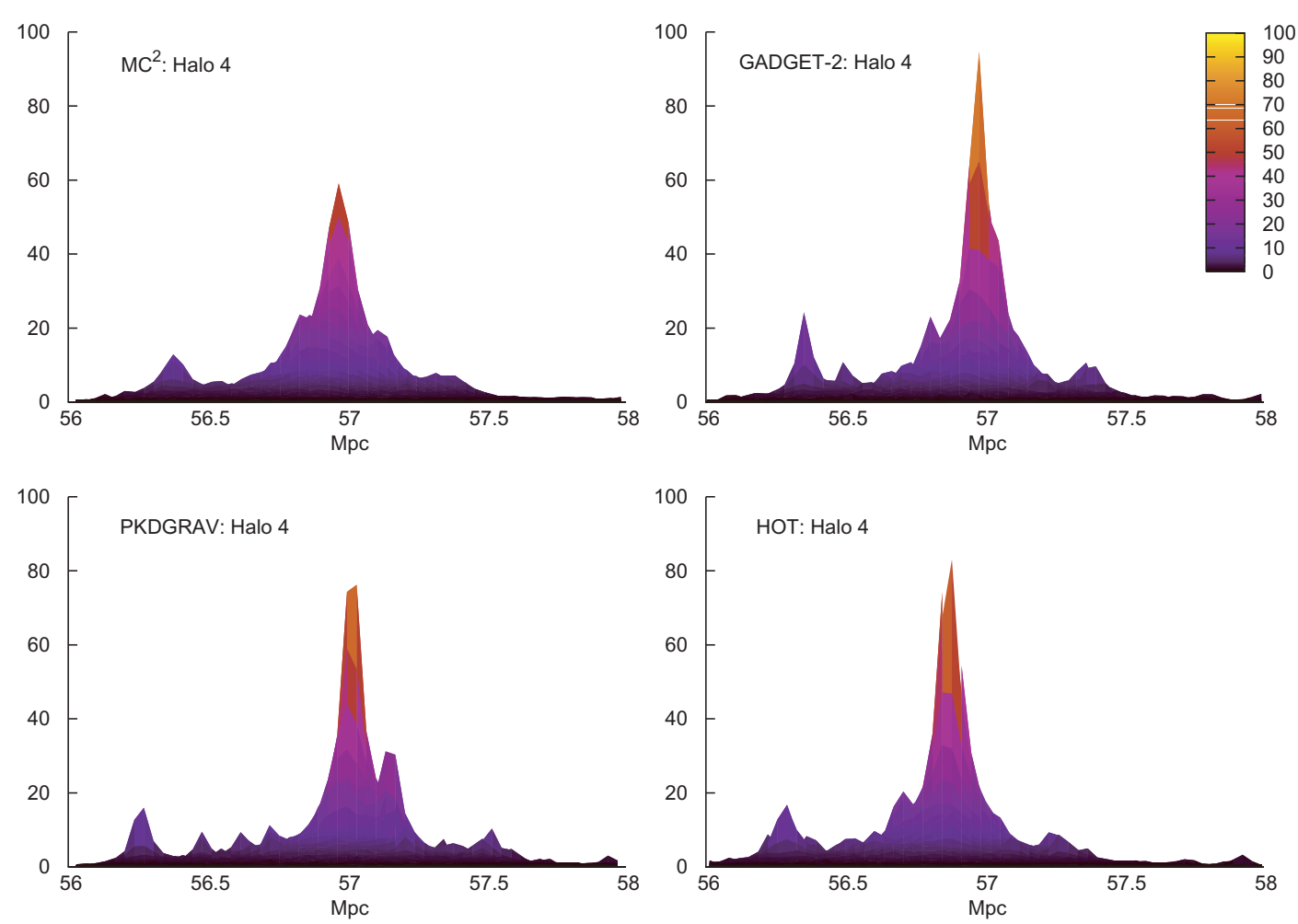

Figure 8. Projected two-dimensional density profile of halo 4 for $\mathrm{MC}^{2}$, GADGET-2, PKDGRAV, and HOT, viewed along the $y$-axis. $\mathrm{MC}^{2}$ shows less substructure and is less dense in the inner region. The profiles were generated following the same procedure as in figure 4.

reasonable (usually the minimum number of particles is several times bigger), but because we are interested in cross-code comparison. We follow the suggestions by Warren et al [52] and correct the halo mass for possible undersampling via:

$$
n_{\mathrm{h}}^{\text {corr }}=n_{\mathrm{h}}\left(1-n_{\mathrm{h}}^{-0.6}\right),
$$

where $n_{\mathrm{h}}$ is the number of particles in a halo. This correction lowers the masses of small mass halos considerably.

In order for small halos to be resolved, both mass and force resolution must be adequate. In [56], resolution criteria for the force resolution are derived:

$$
\frac{\delta_{\mathrm{f}}}{\Delta_{\mathrm{p}}}<0.62\left[\frac{n_{\mathrm{h}} \Omega_{\mathrm{m}}(z)}{\Delta}\right]^{1 / 3},
$$

with $\delta_{\mathrm{f}}$ being the force resolution, $\Delta_{\mathrm{p}}$ being the interparticle spacing, and $\Omega_{\mathrm{m}}(z)$ the matter content of the Universe at a given redshift. Equation (8) predicts that all the non-grid codes have enough force resolution to resolve the smallest halos considered, while the two PM codes, $\mathrm{MC}^{2}$, and PMM, have sufficient force resolution to resolve halos with more than 40 particles, and that the base grid of the two AMR codes restricts them to capturing halos with more than 2500 particles. Of course this is only a rough estimate in principle since the AMR codes increase their local resolution as a function of density threshold, the question is whether the criteria used for this is sufficient to resolve halos starting at 40 particles/halo.

We have indicated the resolution restrictions in figure 9 by vertical lines (dashed: 40 particles, dashed-dotted: 2500 particles). The predictions are good indicators of actual code results. The AMR codes fall off at slightly lower masses than given by 2500 particles. The resolution which determines the smallest halos being captured is apparently being set by the base grid of the AMR codes and not by the highest resolution achieved after refinement. In principle, this is not due to a given choice for the base grid but to the refinement criterion: for the AMR codes to achieve good results, significantly more aggressive density 


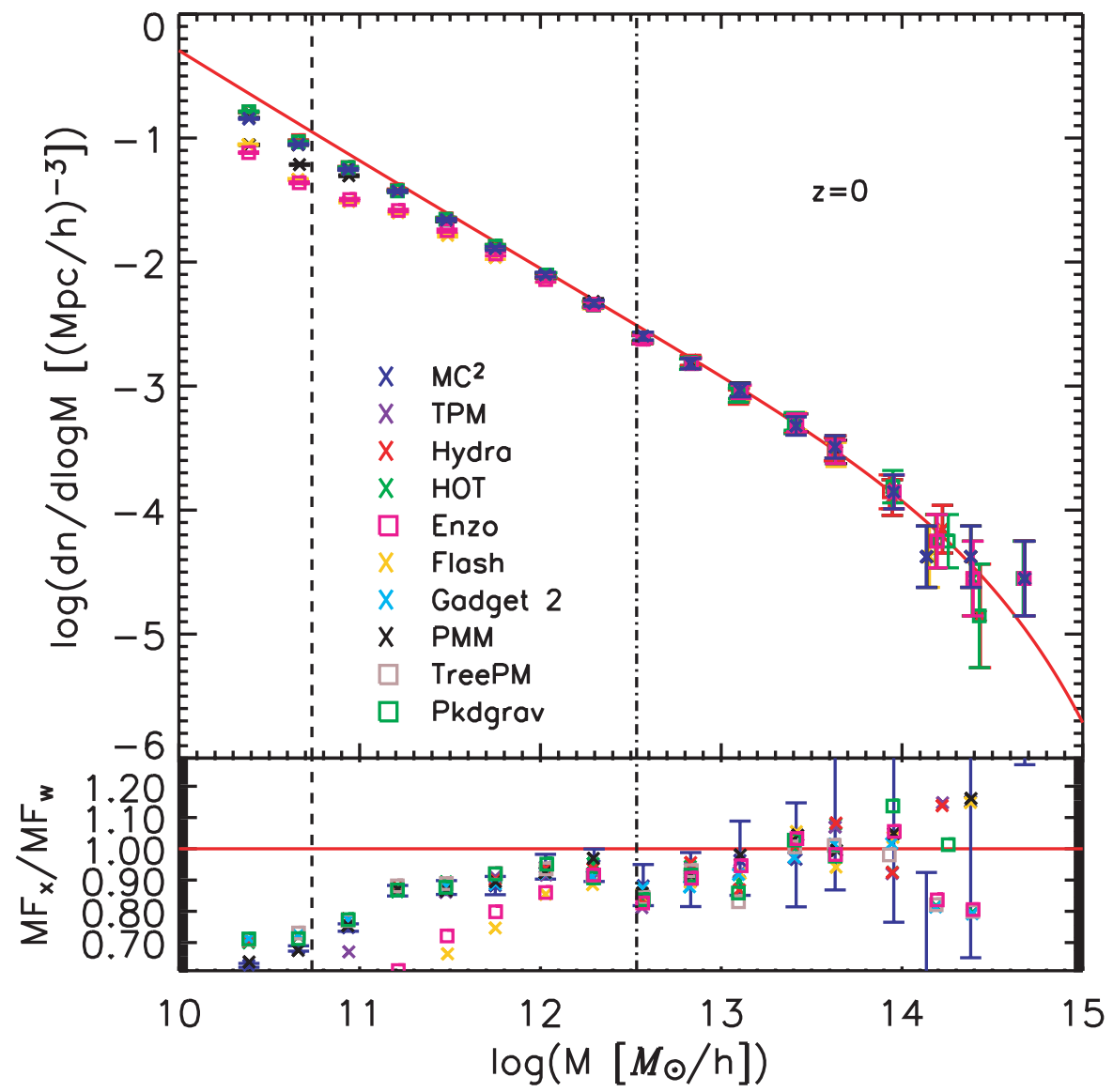

Figure 9. Mass function at $z=0$, simulation results and the Warren fit (red line). Lower panel: residuals with respect to the Warren fit. For clarity, we only show the error bars for one code. The dashed line indicates the threshold for 40 particles (force resolution limit for the PM codes, according to equation (8)), the dotted-dashed line for 2500 particles (force resolution limit for the base grid of the AMR codes).

thresholding appears to be indicated. (Similar results were found in $[2,9]$.$) As predicted, the mass functions$ of the PM codes start to deviate at around 40 particles from the other codes.

Overall the agreement among the codes is very good. For comparison, we show the Warren fit [52] in red. Due to limited statistics imposed by the small box-size, the purpose here is not to check the quality of the fit. At the high mass end, the scatter is as expected due to the rareness of high-mass halos. In the medium mass range between $10^{12.3}$ and $10^{13.4} h^{-1} M_{\odot}$ all codes agree remarkably well, down to the per cent level. In the small halo regime with as low as 40 particles, the agreement of the codes - besides the AMR codes as explained above - stays at this level. This indicates that the halo mass function is a very robust statistic and the simple resolution arguments given above can reliably predict the halo mass limits of the individual simulations.

The comparison yields one surprising result, however: the TPM code simulation has far fewer halos in the regime below 40 particles per halo than the other high resolution codes. This finding was already pointed out in [2]. In order to understand this deficit of halos in more detail, we investigate the halo count as a function of environment in the following.

5.3.2. Halo count and density. In this section, we use ParaView again as the main analysis tool. One very attractive feature of ParaView is a suite of filter functions. These filters allow direct manipulation of the data that is visualized. They include functions such as FFTs, smoothing routines via Gaussian filtering (which we used in the previous section), and tessellation routines, to name a few. We have implemented additional routines to find halos (a fully parallel FOF halo finder integrated into ParaView is under development) and to 

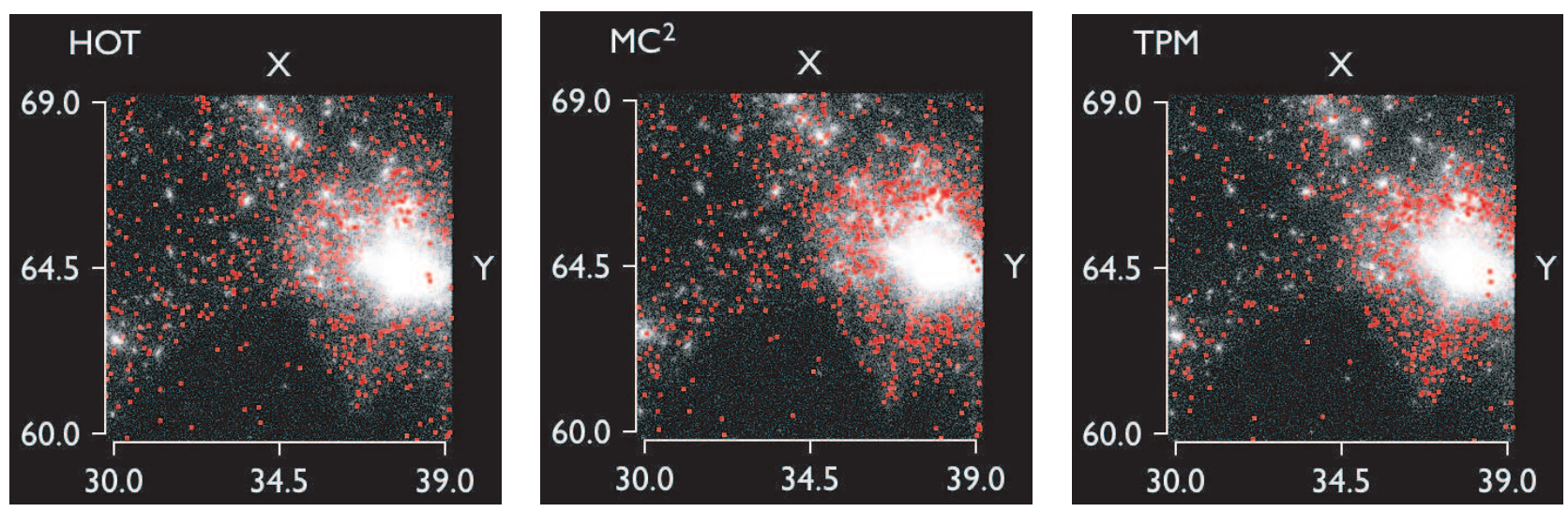

Figure 10. Small halos (10 particles) in the HOT, MC ${ }^{2}$, and TPM simulation. Red points: halos, white dots: subset of the simulation particles. The distribution and number count of the small halos is different in all three codes.

calculate densities from the particle distribution in order to cross-correlate density with halo counts. We have also added an interface to the plotting program Gnuplot. ${ }^{11}$

In the last section, we investigated the mass function and discovered a discrepancy of small halos in the two AMR codes and TPM. The hypothesis for the halo deficit in the AMR codes is, as discussed above, that the refinement criteria were not sufficiently stringent. The resulting too-coarse base grid in the initial state of the simulation suppresses the formation of small halos, and these halos cannot be recovered in the end. This would imply that the AMR simulations should have a deficit of small halos more or less independent of density: small halos should be suppressed everywhere, even in the highest refinement regions. A possible explanation for the missing halos in the TPM simulation could be a hand-over problem between the PM and the tree code. In this case, the number of small halos in high density regions should be correct. A qualitative comparison of three codes (HOT, MC ${ }^{2}$, and TPM) is shown in figure 10. The red points show halos with 10 particles, the white dots are a subset of the simulation particles. It is immediately obvious that the halo counts in different environments, close to the large halo on the right, or on in the lower density regions on the left, are different. After this qualitative result, we have to quantify this finding in order to arrive at a reliable conclusion about the cause for the halo deficits.

We use the VTK toolkit to implement a routine that calculates the density field on a (variable) grid from the particle distribution via a nearest grid point (NGP) algorithm. The grid size for the density field is usually set by the requirement that the density field be not too noisy. As a first check, we compare the density probability distribution function (PDF) for the different codes. It is clear that, if the grid for calculating the density is chosen coarse enough, details should be smoothed out and the PDFs for the different codes should be in good agreement. In figure 11, we show the PDFs for all codes calculated on a $32^{3}$ grid (left panel) corresponding to a smoothing scale of $2 h^{-1} \mathrm{Mpc}$ and a $64^{3}$ grid (right panel) corresponding to a smoothing scale of $1 h^{-1} \mathrm{Mpc}$. In both cases, all codes agree extremely well, as to be expected since the smoothing scales are well beyond the code resolutions. We confirmed that this result holds also for finer grids, up to $256^{3}$, which corresponds to the lowest resolution in the AMR codes Enzo and FLASH. The average number of particles in a grid cell $\bar{\rho}$ on the left panel is 512 particles per cell, in the right panel 64 particles per cell. If we define the density contrast $\delta=(\rho-\bar{\rho}) / \bar{\rho}$ and define void (highly underdense) regions as regions with a density contrast $\delta^{\text {Void }}=-0.8$, we find $\rho^{\text {Void }} \simeq 100$ for the left panel and $\rho^{\text {Void }} \simeq 13$ for the right panel. In both cases, this threshold is on the right of the maximum of the curves - a large fraction of the simulation volume is underdense.

Next, we investigate the correlation of the numbers of halos with density (measured by the number of particles per grid cell on a $32^{3}$ grid). Figure 12 displays the distribution of halos with respect to density for two mass bins: halos with 10-40 particles and halos with 41-2500 particles. These bins were chosen because, as discussed earlier, the force resolution of $\mathrm{MC}^{2}$ and PMM should be sufficient to resolve halos with more than

${ }^{11}$ These new routines are not yet available in the public version of ParaView but we plan to release them in the near future. 

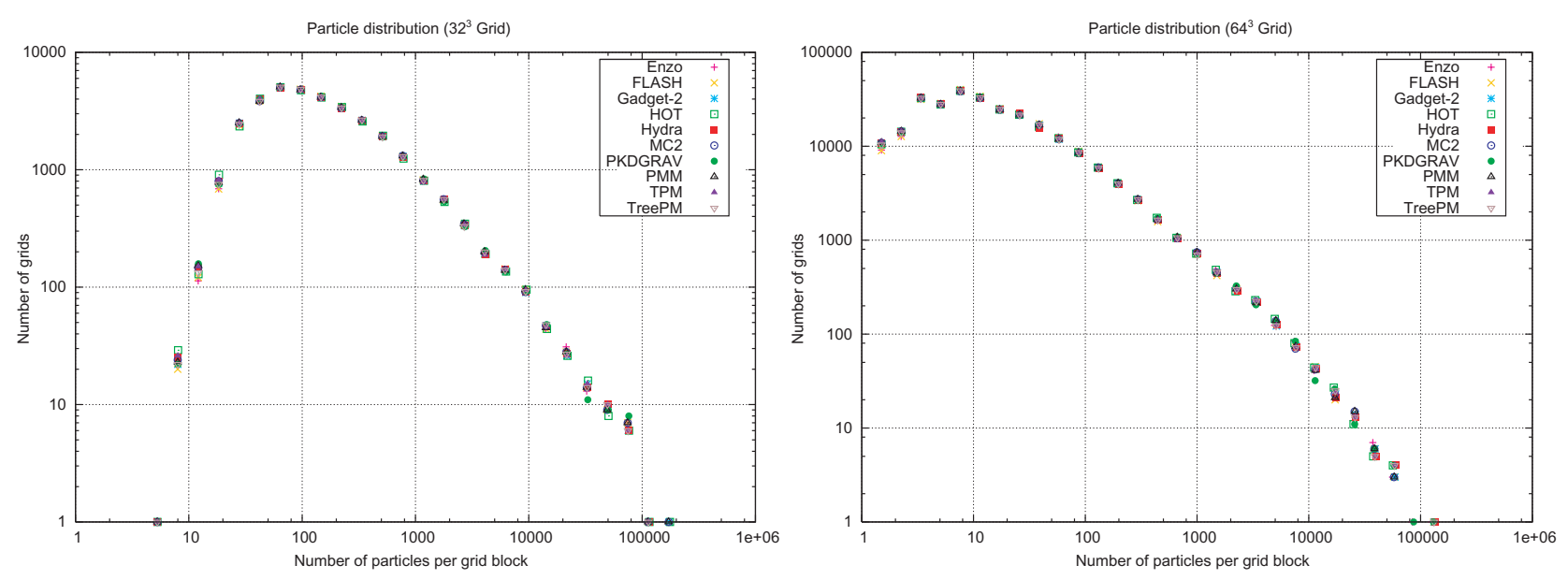

Figure 11. PDF of the densities. Left panel: calculation of the density on a $32^{3}$ grid, right panel: calculation of the density on a $64^{3}$ grid.

40 particles, while Enzo's and FLASH's base grid set this limit to more than 2500 particles, in the absence of refinement. We restrict our investigations to a density threshold of up to 100000 particles per cell which is the density range with the largest halo population.

Figure 12 shows the results for 10-40 particle (left panel) and 41-2500 particle halos (right panel). The lower panels show the residuals with respect to GADGET-2. (We have verified from the mass function, figure 9 that the agreement for larger halos between the ten codes is very good.) The two AMR codes Enzo and FLASH have a deficit for both halo sizes over most of the density region. They only catch up with the other codes at around 10000 particles per cell, in agreement with our previous argument that whether halos are resolvable by the AMR codes or not is being dictated by the size of the base grid. In terms of capturing smaller halos, the refinement only helps in very high density regions.

The result for the TPM simulation is somewhat paradoxical: in the low density region the result for the small halos agrees well with the other high-resolution codes, however, TPM misses a very large number of small halos in the region between 200 and 10000 particles per cell, the curve falling even below the AMR codes. This suggests that the problem of the TPM code is not due to the threshold criterion for the tree but perhaps due to a hand-over problem between the grid and the tree. The two PM codes have slightly lower numbers of very small halos, in good agreement with the prediction that they only resolve halos with more than 40 particles. The agreement between $\mathrm{MC}^{2}$ and PMM itself is excellent. The TreePM code shows a slight excess of small halos compared to the other high-resolution codes. This excess vanishes completely if the cut for the small halos is chosen to be 20 particles instead of 10 particles for the smallest allowed halo. The reason for this difference is not clear, and could lie in the choice of time-integration. The agreement for the medium size halos (left panel) is very good, except for the AMR codes. For the medium size halos, the TPM code again shows a slight deficit of halos in the medium density regime, but far less pronounced than for the small halos. The overall agreement of the high-resolution codes is very good, as is to be expected from the mass function results.

\subsection{The power spectrum}

The matter power spectrum is one of the most important statistics for precision cosmology. Upcoming weak lensing surveys promise measurements of the power spectrum at the $1 \%$ accuracy level out to length scales of $k \sim 10 \mathrm{~h} \mathrm{Mpc}^{-1}$ (for an overview of the requirements for the accuracy of predictions for future lensing surveys, see, e.g., [59]). This poses a severe theoretical challenge: predicting the matter power spectrum at the same level of accuracy. A first step for showing that this is possible is to investigate how well the matter power spectrum can be predicted from pure dark matter simulations, baryonic physics being included as a second step. It has already been shown that at the length scales of interest, hydrodynamic effects can alter the matter power spectrum at up to $10 \%$ [60]. In this paper, we concentrate on the first step and determine how well a 

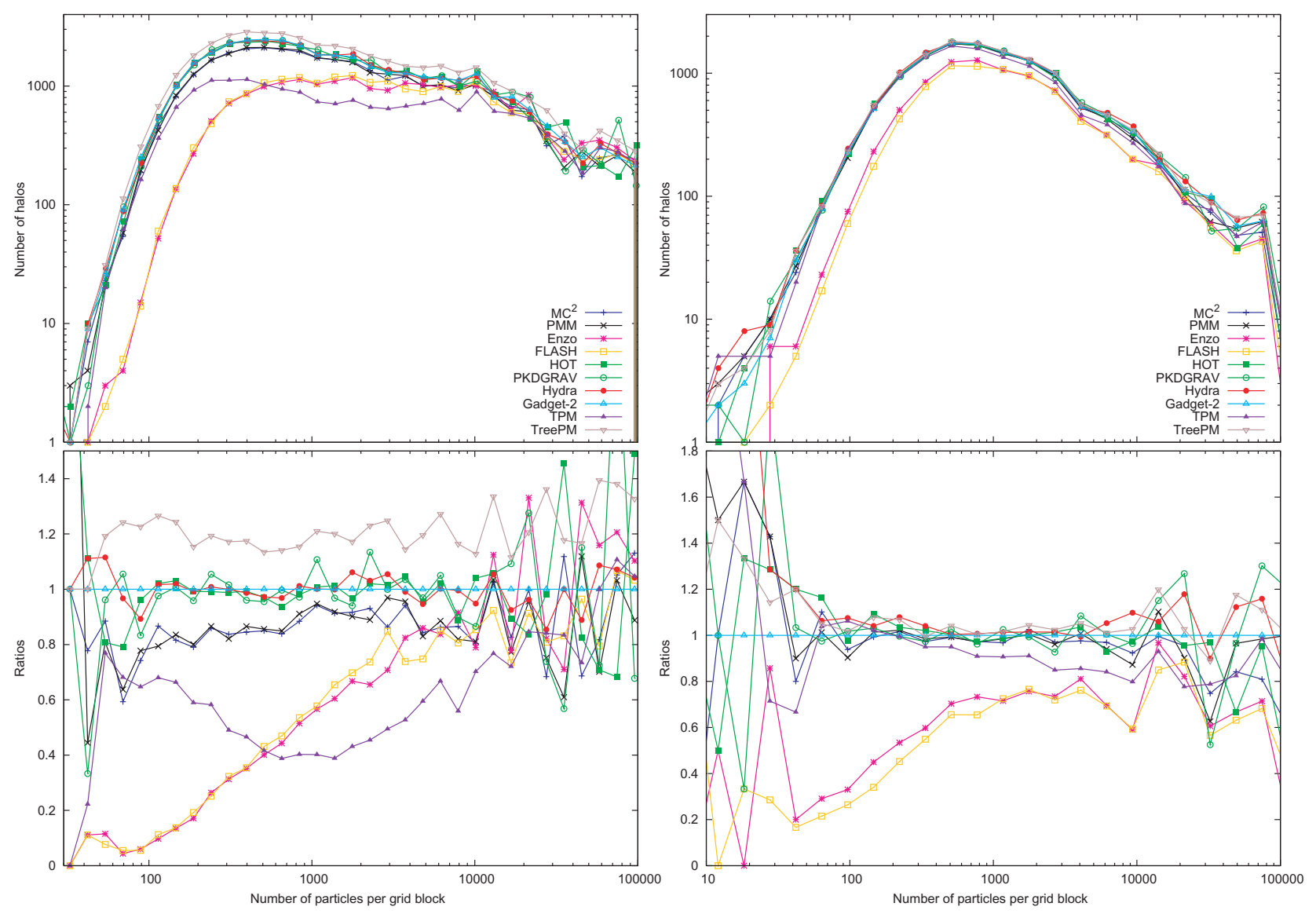

Figure 12. Number of halos as a function of density. Left panel: halos with 10-40 particles, right panel: halos with 41-2500 particles. The lower panels show the residuals with respect to GADGET-2. Both panels show the deficit of small halos in Enzo and FLASH over most of the density region —only at very high densities do the results catch up. The behavior of the TPM simulation is interesting: not only does this simulation have a deficit of small halos but also the deficit is very significant in medium density regions, in fact falling below the two AMR codes. The slight excess of small halos shown in the TreePM run vanishes completely if the halo cut is raised to 20 particles per halo and the TreePM results are in that case in excellent agreement with GADGET-2.

diverse set of $\mathrm{N}$-body codes agree with each other for the prediction of the matter power spectrum. In future work, we aim to predict the dark matter power spectrum at $k \sim 1 h \mathrm{Mpc}^{-1}$ at the level of $1 \%$ precision or better. This will include a detailed analysis of the accuracy of the initial conditions, and convergence studies of the nonlinear evolution including comparisons against perturbation theory, a task beyond the scope of the current paper.

We determine the matter power spectrum by generating the density field from the particles via a cloud-in-cell (CIC) routine on a $1024^{3}$ spatial grid and then obtain the density in $k$-space by applying a $1024^{3}$ FFT. The square of the $k$-space density yields the power spectrum: $P(k)=\left\langle\left|\delta(k)^{2}\right|\right\rangle$. The CIC routine introduces a filter at small length scale. We compensate for this filtering artifact by deconvolving the $k$-space density with a CIC window function.

The results for the different codes are shown in figure 13. Note that the box size of $64 h^{-1} \mathrm{Mpc}$ is too small for a realistic cosmological power spectrum calculation, as the largest modes in the box no longer evolve linearly. This leads to an incorrect onset of the nonlinear turn-over in the power spectrum. Nevertheless, the comparison of the different codes is very informative. The upper panel in figure 13 shows the results for the power spectra themselves. The lower resolution of the grid codes is very apparent, their results falling away at $k \sim 2 h \mathrm{Mpc}^{-1}$. The middle panel shows the residuals of all codes with respect to GADGET-2. All codes agree at roughly $1 \%$ out to $k \sim 1 h \mathrm{Mpc}^{-1}$. PKDGRAV shows small scatter in the linear regime. This might 


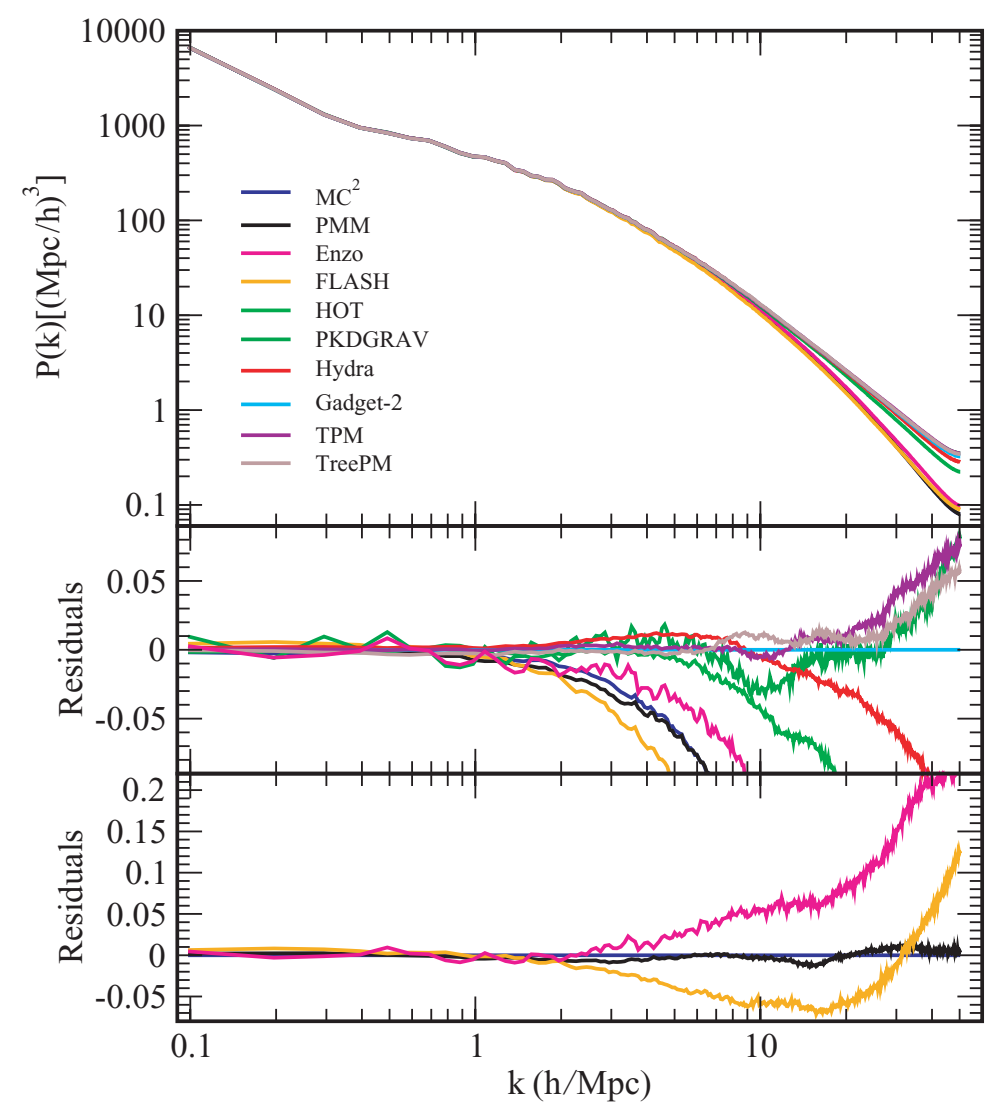

Figure 13. Power spectrum results and the residuals for the different codes. Upper panel: comparison of the different power spectra. Middle panel: residuals of all codes with respect to GADGET-2. Lower panel: residuals of the mesh codes with respect to $\mathrm{MC}^{2}$.

be caused by imprecise periodic boundary conditions, which are not as easy to implement in tree codes as they are for grid codes. The high-resolution codes agree to better than $5 \%$ out to $k \sim 10 \mathrm{hpc}^{-1}$. At that point HOT and Hydra lose power, while PKDGRAV, TPM and TreePM show slightly enhanced power compared to the GADGET-2 run. The formal force resolutions of the codes would suggest that the different runs (including the grid runs) should agree much better at the wavenumbers shown.

The $1024^{3}$ FFT used to generate the power spectra is far below the resolution of the non-grid codes and at the resolution limit of the AMR and PM codes. The discrepancy might be due to several reasons: the number of time steps, the accuracy of the force solvers, the accuracy of reaching $z=0$ at the end of each run, just to suggest a few. A more detailed study of the power spectrum including larger simulation boxes is certainly required for application to upcoming surveys. In the lower panel, we show a comparison of the grid codes only, with respect to $\mathrm{MC}^{2}$. The two pure PM codes, $\mathrm{MC}^{2}$, and PMM agree remarkably well over the whole $k$-range under consideration, the difference being below 1\%. The two AMR codes, Flash and Enzo, deviate considerably, most likely due to different refinement criteria. It is somewhat surprising that Enzo has larger power than the two PM codes, which have the same resolution in the whole box that Enzo has only in high density regions. This could be the result of an algorithmic artifact in the AMR implementation.

To summarize, the agreement for the matter power spectrum is at the $5 \%$ level over a large range in length scale. The early deviation of the grid codes is surprising, as the nominal resolution of all codes should have been sufficient to generate agreement over a wider $k$-range. In order to be able to obtain more cosmologically relevant results at $k \sim 1 h \mathrm{Mpc}^{-1}$, much larger simulation boxes have to be compared. At higher wavenumbers, baryonic effects become important leading to the necessity of a much more involved comparison setup. 


\section{Discussion and conclusion}

The new era of precision cosmology requires new standards for the dynamic range and accuracy of large cosmological simulations. While previously, qualitative answers and quantitative results at the $20 \%$ accuracy level were sufficient, we now need to robustly predict nonlinear physics at the $1 \%$ accuracy level. More stringent levels of code verification and error control are but the first step in this demanding task.

In this paper, we have carried out a comprehensive code comparison project with 10 state-of-the-art cosmological simulation codes. ParaView was introduced as a powerful analysis tool which should make it more convenient for code developers to compare results. In particular, results from the current suite of simulations can function as a good database for reference purposes and benchmarking. The initial conditions are publicly available ${ }^{12}$. We are further strengthening this project by integrating a provenance system that logs information regarding how data came into being and how it was processed [61].

We find that the halo mass function is a very stable statistic, the agreement over wide ranges of mass being better than $5 \%$. Additionally, the low mass cutoff for individual codes can be reliably predicted by a simple criterion. The internal structure of halos in the outer regions of $\sim R_{200}$ also appears to be very similar between different simulation codes. Larger differences between the codes in the inner region of the halos occur if the halo is not in a relaxed state: in this case, time stepping issues might also play an important role (e.g. particle orbit phase errors, global time mismatches). For halos with a clear single center, the agreement is very good and predictions for the fall-off of the profiles from resolution criteria hold as expected. The investigation of the halo counts as a function of density revealed an interesting problem with the TPM code, the simulation suffering from a large deficit in medium density regimes. The AMR codes showed a large deficit of small halos over almost the entire density regime, as the (insufficiently refined) base grid of the AMR simulation set too low a resolution limit for the halos.

The power spectrum measurements revealed definitely more scatter among the different codes than expected. The agreement in the nonlinear regime is at the 5-10\% level, even on moderate spatial scales around $k=10 \mathrm{hpc}^{-1}$. This disagreement on small scales is connected to differences of the codes in the inner regions of the halos.

The results from the code comparisons are satisfactory and not unexpected, but also show that much more work is needed in order to meet the simulation challenge for upcoming surveys. While this paper does not directly address the problem of calibration (proving high accuracy results), it verifies the robustness of code results over a wide range of cosmological statistics at the $10 \%$ level.

In order to reach sub-per cent levels of accuracy for a broad range of observables it is very unlikely that a single strategy will suffice; codes will have to be tuned in very specific ways to address the problem at hand. For example, small-scale structure will require very high mass resolution and a corresponding focus on time-stepping errors, whereas high accuracy on large length scales will require paying close attention to accurate initial conditions and control of force errors at modest wavenumbers. Additionally, in several cases, baryonic effects enter at the same level as $N$-body errors, as is the case for weak lensing observations on scales of $k>1 h \mathrm{Mpc}^{-1}$. To overcome this hurdle, self-calibration from observations may have to be employed aside from the addition of brute-force hydro-solvers (which have their own errors and physics uncertainties).

Convergence tests on idealized and real-world problems are extremely valuable tools, however there are instances where codes can converge to an incorrect answer (for some extraneous reason such as a finite-size limitation) or simply stop converging beyond a certain point. Thus, the use of multiple codes will remain an important tool to establish confidence in the validity of numerical results.

\section{Acknowledgments}

The calculations described herein were performed in part using the computational resources of Los Alamos National Laboratory. A special acknowledgment is due to supercomputing time awarded to us under the LANL Institutional Computing Initiative.

\footnotetext{
12 http://t8web.lanl.gov/people/heitmann/arxiv
} 


\section{References}

[1] Zel'dovich Y B 1970 Astron. Astrophys. 584 Shandarin S and Zel'dovich Y B 1989 Rev. Mod. Phys. 61185

[2] Heitmann K, Ricker P M, Warren M S and Habib S 2005 Astrophys. J. Suppl. 16028

[3] Ryne R D et al 1998 The US DOE Grand Challenge in Computational Accelerator Physics, Proc. LINAC98 (Chicago, IL)

[4] Qiang J, Ryne R D, Habib S and Decyk V 2000 J. Comput. Phys. 163434

[5] Trac H and Pen U-L 2006 New Astron. 11273

[6] Bryan G L and Norman M L 1996 12th Kingston Meeting on Theoretical Astrophysics, Proc. of Meeting Held in Halifax; Nova Scotia (ASP Conference Series vol 123) ed D A Clarke and M Fall (San Francisco, CA: Astronomical Society of the Pacific)

[7] Bryan G L and Norman M L 1999 Workshop on Structured Adaptive Mesh Refinement Grid Methods (IMA Volumes in Mathematics Vol 117) ed N Chrisochoides (Berlin: Springer)

[8] O'Shea B W, Bryan G, Bordner J, Norman M L, Abel T, Harkness R and Kritsuk A 2004 Adaptive Mesh Refinement-Theory and Applications ed T Plewa, T Linde and G Weirs (Berlin: Springer)

[9] O’Shea B W, Nagamine K, Springel V, Hernquist L and Norman M L 2005 Astrophys. J. Suppl. 1601

[10] Berger M J and Colella P 1989 J. Comput. Phys. 8264

[11] Efstathiou G, Davis M, White S D M and Frenk C S 1985 Astrophys. J. Suppl. 57241

[12] Hockney R W and Eastwood J W 1988 Computer Simulation Using Particles (Bristol: Institute of Physics Publishing)

[13] Colella P and Woodward P R 1984 J. Comput. Phys. 54174

[14] Bryan G L, Norman M L, Stone J M, Cen R and Ostriker J P 1995 Comput. Phys. Commun. 89149

[15] Fryxell B et al 2000 Astrophys. J. Suppl. 131273

[16] MacNeice P, Olson K M, Mobarry C, de Fainchtein R and Packer C 2000 Comput. Phys. Commun. 126330

[17] Quirk J J 1991 PhD Thesis Cranfield Institute of Technology

[18] de Zeeuw D and Powell K G 1993 J. Comput. Phys. 10456

[19] Warren M S and Salmon J K 1993 Supercomputing '93 (Los Alamitos, CA: IEEE Computer Society Press) p 12

[20] Salmon J K and Warren M S 1994 J. Comput. Phys. 111136

[21] Warren M S, Fryer C L and Goda M P 2003 Proc. ACM/IEEE SC2003 Conf. (New York: ACM Press)

[22] Frenk C S et al 1999 Astrophys. J. 525554

[23] Fryer C L and Warren M S 2002 Astrophys. J. 574 L65

[24] Dikaiakos M D and Stadel J 1996 ICS Conf. Proc.

[25] Barnes J and Hut P 1986 Nature 324446

[26] Hernquist L, Bouchet F and Suto Y 1991 Astrophys. J. Suppl. 75231

[27] Couchman H M P 1999 J. Comput. Appl. Math. 109373

[28] Springel V, Yoshida N and White S D M 2001 New Astron. 679

[29] Springel V 2005 Mon. Not. R. Astron. Soc. 3641105

[30] Bagla J S 2002 J. Astrophys. Astron. 23185

[31] Monaghan J J 1992 Annu. Rev. Astron. Astrophys. 30543

[32] Springel V and Hernquist L 2002 Mon. Not. R. Astron. Soc. 333649

[33] Xu G 1995 Astrophys. J. Suppl. 98355

[34] Bode P, Ostriker J P and Xu G 2000 Astrophys. J. Suppl. 128561

[35] White M 2002 Astrophys. J. Suppl. 143241

[36] Klypin A A and Holtzman J 1997 Preprint astro-ph/9712217

[37] Bardeen J M, Bond J R, Kaiser N and Szalay A S 1986 Astrophys. J. 30415

[38] Dehnen W 2001 Mon. Not. R. Astron. Soc. 324273

[39] Ahrens J, Geveci B and Law C 2005 ParaView: an end-user tool for large data visualization The Visualization Handbook ed C Hansen and C Johnson (New York: Academic) pp 717-31

[40] Lacey C and Cole S 1994 Mon. Not. R. Astron. Soc. 271676

[41] Davis M, Efstathiou G, Frenk C S and White S D M 1985 Astrophys. J. 292371

[42] Navarro J F, Frenk C S and White S D M 1995 Mon. Not. R. Astron. Soc. 267401

[43] Bullock J S et al 2001 Mon. Not. R. Astron. Soc. 321559

[44] White M 2001 Astron. Astrophys. 36727

[45] Lukić Z, Reed D, Habib S and Heitmann K 2008 Preprint arXiv:0803.3624

[46] Majumdar S and Mohr J J 2004 Astrophys. J. 61341 
[47] Press W H and Schechter P 1974 Astrophys. J. 187425

[48] Lee J and Shandarin S 1998 Astrophys. J. 50014

[49] Sheth R K, Mo H J and Tormen G 2001 Mon. Not. R. Astron. Soc. 3231

[50] Sheth R K and Tormen G 1999 Mon. Not. R. Astron. Soc. 308119

[51] Jenkins A et al 2001 Mon. Not. R. Astron. Soc. 321372

[52] Warren M S, Abazajian K, Holz D E and Teodoro L 2006 Astrophys. J. 646881

[53] Reed D et al 2003 Mon. Not. R. Astron. Soc. 346565

[54] Reed D, Bower R, Frenk C, Jenkins A and Theuns T 2007 Mon. Not. R. Astron. Soc. 3742

[55] Heitmann K, Lukić Z, Habib S and Ricker P M 2006 Astrophys. J. 642 L85

[56] Lukić Z, Heitmann K, Habib S, Bashinsky S and Ricker P M 2007 Astrophys. J. 6711160

[57] Barkana R and Loeb A 2004 Astrophys. J. 609474

[58] Bagla J S and Prasad J 2006 Mon. Not. R. Astron. Soc. 370993

[59] Huterer D and Takada M 2005 Astropart. Phys. 23369

[60] Zhan H and Knox L 2004 Astrophys. Lett. 616 L75

White M 2004 Astropart. Phys. 22211

Jing Y P, Zhang P, Lin W P, Gao L and Springel V 2006 Astrophys. Lett. 640119

Rudd D H, Zentner A R and Kravtsov A 2008 Astrophys. J. 67219

[61] Anderson E W, Silva C T, Ahrens J P, Heitmann K and Habib S 2008 Comput. Sci. Eng. 1030 